HRI-P-08-02-001

CU-PHYSICS/03-2008

\title{
Optimizing the greenfield Beta-beam
}

\author{
Sanjib Kumar Agarwalla ${ }^{\star, \dagger}$, Sandhya Choubey ${ }^{\star}$, \\ Amitava Raychaudhuri ${ }^{\star, \dagger}$, Walter Winter ${ }^{\sharp}$ \\ ${ }^{\star}$ Harish-Chandra Research Institute, \\ Chhatnag Road, Jhunsi, Allahabad 211019, India \\ ${ }^{\dagger}$ Department of Physics, University of Calcutta, \\ 92 Acharya Prafulla Chandra Road, Kolkata 700009, India \\ ${ }^{\sharp}$ Institut für Theoretische Physik und Astrophysik, Universität Würzburg, \\ Am Hubland, D-97074 Würzburg, Germany
}

\begin{abstract}
We perform a comprehensive and detailed comparison of the physics reach of Beta-beam experiments between two pairs of plausible source ions, $\left({ }^{8} \mathrm{~B},{ }^{8} \mathrm{Li}\right)$ and $\left({ }^{18} \mathrm{Ne},{ }^{6} \mathrm{He}\right)$. We study the optimal choices for the baseline, boost factor, and luminosity. We take a 50 kton iron calorimeter, a la ICAL@INO, as the far detector. We follow two complementary approaches for our study: (i) Fixing the number of useful ion decays and boost factor of the beam, and optimizing for the sensitivity reach between the two pairs of ions as a function of the baseline. (ii) Matching the shape of the spectrum between the two pairs of ions, and studying the requirements for baseline, boost factor, and luminosity. We find that for each pair of ions there are two baselines with very good sensitivity reaches: a short baseline with $L[\mathrm{~km}] / \gamma \simeq 2.6\left({ }^{8} \mathrm{~B}+{ }^{8} \mathrm{Li}\right)$ and $L[\mathrm{~km}] / \gamma \simeq 0.8$ $\left({ }^{18} \mathrm{Ne}+{ }^{6} \mathrm{He}\right)$, respectively, and a long "magic" baseline. For $\gamma \sim 500$, one would optimally use ${ }^{6} \mathrm{He}$ and ${ }^{18} \mathrm{Ne}$ at the short baseline for $\mathrm{CP}$ violation, ${ }^{8} \mathrm{~B}$ and ${ }^{8} \mathrm{Li}$ at the magic baseline for the mass hierarchy, and either ${ }^{6} \mathrm{He}$ and ${ }^{18} \mathrm{Ne}$ at the short baseline or ${ }^{8} \mathrm{~B}$ and ${ }^{8} \mathrm{Li}$ at the magic baseline for the $\sin ^{2} 2 \theta_{13}$ discovery.
\end{abstract}

${ }^{a}$ email: sanjib@hri.res.in

${ }^{b}$ email: sandhya@hri.res.in

${ }^{c}$ email: raychaud@hri.res.in

${ }^{d}$ email: winter@physik.uni-wuerzburg.de 


\section{Introduction}

Spectacular results from a series of neutrino oscillation experiments over the last four decades [1, $2,3,4,5,6,7]$ have paved the way for the "golden" age of neutrino physics. Determining the hitherto unknown mixing angle $\theta_{13}$, the $\mathrm{CP}$ phase $\delta_{\mathrm{CP}}$, and the sign of $\Delta m_{31}^{2}$, i.e., the neutrino mass hierarchy ${ }^{1}$, have emerged as the next frontier in this field. All these three quantities can be probed by experimentally measuring the so-called "golden" channel [8] oscillation probability $P_{e \mu}$ (or its T-conjugate channel $P_{\mu e}$ ). A series of ambitious projects are under discussion which plan to use this oscillation channel. The on-going and near future experiments include the MINOS experiment in the US [6], and the CNGS experiments ICARUS [9] and OPERA [10] in Europe. Next experiments in line will be T2K in Japan [11] and $\mathrm{NO} \nu \mathrm{A}$ in US [12]. All these experiments will use muon neutrino beams from conventional accelerator sources in order to observe $P_{\mu e}$. Collectively and in combination with short-baseline reactor experiments, such as Double Chooz [13], these experiments are expected to improve the bound on $\theta_{13}$ to about $\sin ^{2} 2 \theta_{13}<0.01(90 \%$ CL) [14]. The mass hierarchy and CP violation, though in principle accessible using the combined data from the $\mathrm{T} 2 \mathrm{~K}$ and $\mathrm{NO} \nu \mathrm{A}$ experiments, can be determined only for values of $\sin ^{2} 2 \theta_{13}$ (true) close to the current bound and for some fraction of the possible values of the CP phase $\delta_{\mathrm{CP}}\left(\right.$ true).$^{2}$ The sensitivity of these experiments is mainly restricted by statistics, while for larger luminosity setups, the intrinsic $\nu_{e}$ background poses a natural limitation for experiments sensitive to $\nu_{\mu}$ oscillations into $\nu_{e}$. Therefore, if Nature has not been very kind we will need larger experiments to complete our understanding of the neutrinos, possibly using an alternate technology.

In order to access small values of $\sin ^{2} 2 \theta_{13}$, there are several requirements: One needs to have low backgrounds, one needs more statistics, and one needs to reduce systematical uncertainties. As far as the low background requirement is concerned, it is an advantage to use a pure flavor neutrino beam without any intrinsic beam contamination. One such approach has been proposed by Piero Zucchelli [15]: Radioactive nuclides are created by impinging a target by accelerated protons. These unstable nuclides are collected, fully ionized, bunched, accelerated and then stored in a decay ring (see for e.g. $[16,17]$ ). The decay of these highly boosted ions in the straight sections of the decay ring produces the so-called Beta-beam. An alternative approach is the socalled neutrino factory (NuFact) [18]. It involves producing, collecting, cooling, accelerating, and circulating muon packets in a storage ring. The decay of accelerated muons (antimuons) in the straight sections of the storage rings produce $\nu_{\mu}$ and $\bar{\nu}_{e}\left(\bar{\nu}_{\mu}\right.$ and $\left.\nu_{e}\right)$ beams. The presence of the $\bar{\nu}_{e}\left(\nu_{e}\right)$ in the beam allows for the observation of the $P_{\bar{e} \bar{\mu}}\left(P_{e \mu}\right)$ oscillation probability in the far detector. Since both $\nu_{\mu}$ (or $\left.\bar{\nu}_{\mu}\right)$ from the original beam as well as $\bar{\nu}_{\mu}\left(\right.$ or $\left.\nu_{\mu}\right)$ from the oscillated $\bar{\nu}_{e}$ (or $\nu_{e}$ ) will be arriving at the detector, it must have the ability to distinguish one from the other. The most accepted candidate is the magnetized iron detector, though there are several proposals with more expensive and elaborate designs and therefore better performance [19]. Statistics can be increased by a higher beam power and the size and efficiency of the detector. Beam-related systematic uncertainties can be reduced to a large extent by working with a two detector set-

\footnotetext{
${ }^{1}$ Though we call this the neutrino mass hierarchy, what we mean is basically the ordering of the neutrino mass states. Therefore, our discussions are valid for both hierarchical as well as quasi-degenerate mass spectra.

${ }^{2}$ We distinguish between the "true" values of the oscillation parameters, which are the values chosen by Nature, and their fitted values. Throughout this paper we denote the true value of a parameter by putting "(true)" after the symbol for the parameter.
} 
up, one very close to the beam line and another serving as the far detector. The systematic uncertainties coming from the lack of knowledge of the neutrino-nucleus interaction cross-sections are another important source of error. These can be controlled to some degree by the near-far two detector set-up, but they cannot be canceled completely [20]. Beam-related backgrounds are extremely small for the NuFact and Beta-beam experiments because they either use leptonic decays (NuFact) or a flavor-pure beam (Beta-beam). The detector backgrounds coming mainly from neutral current interactions and mis-identification of particles, can be reduced by imposing intelligent cuts. The atmospheric neutrino backgrounds, which can be important for Beta-beams at lower energies, can be suppressed using timing and directional information.

The performance and physics reach of these expensive and ambitious experiments have been the subject of much discussion for the past few years [21]. There has been a plethora of papers on this issue $e^{3}$, most of which have addressed the problem of "parameter degeneracies". Even if both neutrinos and antineutrinos are used, there are three types of discrete degeneracies in the golden channel:

1. the $\left(\theta_{13}, \delta_{\mathrm{CP}}\right)$ intrinsic degeneracy [23],

2. the $\left(\operatorname{sgn}\left(\Delta \mathrm{m}_{31}^{2}\right), \delta_{\mathrm{CP}}\right)$ degeneracy [24],

3. the $\left(\theta_{23}, \pi / 2-\theta_{23}\right)$ degeneracy [25].

Together they can result in up to eight-fold degenerate solutions [26], severely deteriorating the sensitivity of the experiment. The variety of suggestions to solve this problem includes combining data from several experiments observing the golden channel, but with different baselines $L$ and neutrino energies $E[23,27,28]$, combining data from accelerator experiments observing different oscillation channels $[29,30,31]$, combining the golden channel data with those from atmospheric neutrino $[32,33]$ or reactor antineutrino experiments [34]. A particularly attractive way of completely resolving at least two of the three degeneracies is to perform the experiment at the "magic baseline" $[35,36,37]$. This magic baseline reflects the characteristic oscillation wavelength corresponding to Earth matter. One can show that at this baseline, for reasonably small values of $\theta_{13}$, the $\delta_{\mathrm{CP}}$-dependent terms vanish. The $\delta_{\mathrm{CP}}$-dependence is therefore reduced and one can eliminate the $\left(\theta_{13}, \delta_{\mathrm{CP}}\right)$ and $\left(\operatorname{sgn}\left(\Delta \mathrm{m}_{31}^{2}\right), \delta_{\mathrm{CP}}\right)$ degeneracies, resulting in tremendous sensitivity to $\theta_{13}$ and $\operatorname{sgn}\left(\Delta \mathrm{m}_{31}^{2}\right)$. The sensitivity reach of a NuFact experiment at the magic baseline can be found in $[35,38]$. The idea for a magic baseline Beta-beam experiment with a similar performance for $\theta_{13}$ and $\operatorname{sgn}\left(\Delta \mathrm{m}_{31}^{2}\right)$ was put forth in Refs. [39, 40, 41].

The sensitivity reach of an experiment depends crucially on beam, baseline, and detector properties. It is therefore important to ask which beam, baseline and detector set-up would qualify as the optimal choice in order to obtain the best $\sin ^{2} 2 \theta_{13}$ reaches for the three quantities we have set out to measure, i.e., $\theta_{13}, \operatorname{sgn}\left(\Delta \mathrm{m}_{31}^{2}\right)$, and $\delta_{\mathrm{CP}}$. For the NuFact, this detailed exercise was performed in Ref. [38]. The sensitivity to each of the three parameters mentioned above was studied as a function of the baseline and muon neutrino energy. It was demonstrated that the minimal muon neutrino energy acceptable for the magnetized iron detector was about $20 \mathrm{GeV}$.

\footnotetext{
${ }^{3} \mathrm{~A}$ summary of the potential of selected NuFact and Beta-beam set-ups have been compiled by the physics working group of the International Scoping Study for a future Neutrino factory, Superbeam and Beta-beam, in their report [22].
} 
The optimal baseline for probing $\theta_{13}$ and $\operatorname{sgn}\left(\Delta \mathrm{m}_{31}^{2}\right)$ is the magic baseline, whereas best sensitivity to $\mathrm{CP}$ violation is expected at $L \sim 3000$ to $5000 \mathrm{~km}[38]$.

For Beta-beams, a variety of plausible set-ups have been proposed in the literature [33, 39, 40, 41, 42, 43, 44, 45, 46, 47, 48, 49, 50, 51, 52] The most popular proposal, which poses minimal challenge for the Beta-beam design, is the commonly called CERN-MEMPHYS project [33, 42, 43]. It proposes to use the EURISOL ion source to produce the radioactive source ions ${ }^{18} \mathrm{Ne}$ and ${ }^{6} \mathrm{He}$, and demands a Lorentz boost factor $\gamma \simeq 100$ for them, which can be produced using the existing accelerator facilities at CERN. The far detector MEMPHYS, a megaton water detector with fiducial mass of 440 kton, will have to be built in the Fréjus tunnel, at a distance of $130 \mathrm{~km}$ from CERN. A high performance set-up proposed in [39, 40, 41] would use a high $\gamma$ Beta-beam and a magnetized iron detector placed at a distance close to the magic baseline. Here one needs to employ an alternative set of source ions, ${ }^{8} \mathrm{~B}$ and ${ }^{8} \mathrm{Li}[53,54]$, with a $\gamma$ between 350 and 650 . As one possible option, the Beta-beam could be targeted from CERN towards the India-Based Neutrino Observatory (INO) [55]. The CERN to INO distance corresponds to $7152 \mathrm{~km}$, which is almost magic. Therefore, this experiment yields sensitivity to $\theta_{13}$ and $\operatorname{sgn}\left(\Delta \mathrm{m}_{31}^{2}\right)$, which can only be outperformed by the NuFact experiment at the magic baseline distance. Set-ups with a neutrino beam from CERN to GranSasso or CanFranc [44] and from Fermilab [47] ( $L \sim 300 \mathrm{~km})$ have also been proposed, and their sensitivity reach has been explored. A comparison of the physics reach among different Beta-beam experimental proposals can be found in Ref. [56].

Higher gamma Beta-beam options have been studied in Refs. [46, 48]. In contrast to earlier works, we study the baseline optimization as a function of the ion pair used $\left({ }^{18} \mathrm{Ne}+{ }^{6} \mathrm{He}\right.$ or $\left.{ }^{8} \mathrm{~B}+{ }^{8} \mathrm{Li}\right)$, and we discuss the impact of the luminosity. In addition, we perform a simultaneous optimization of $L$ and $\gamma$. All comparisons are performed for the same detector, which is a 50 kton magnetized iron calorimeter. Note that the magnetization of the detector, which is mandatory for the neutrino factory, is only used for a reduction of the backgrounds. We study the performance with respect to $\theta_{13}, \operatorname{sgn}\left(\Delta \mathrm{m}_{31}^{2}\right)$, and $\mathrm{CP}$ violation. paper is organized as follows. We describe the Beta-beam experiment and our analysis procedure in Section 2. In Section 3, we optimize the baseline $L$ of the experiment for fixed sets of $\gamma$ 's. In Section 4, we then perform a simultaneous optimization over $L$ and $\gamma$, and we discuss the requirements for a similar beam spectrum. In Section 5, we then show the impact of the Beta-beam luminosity, and determine the best combination of $L$, $\gamma$, and $N_{\beta}$ (the number of useful ion decays per year). Our conclusions can be found in Section 6 .

\section{Simulation of the Beta-beam Experiment}

Here we describe the experimental setup for our proposed Beta-beam facility. We give the details of the flux and detector set-up we have used in our analysis.

\subsection{The Flux}

A Beta-beam [15] is an intense and highly collimated source of pure $\nu_{e}$ or $\bar{\nu}_{e}$ flux, produced from the decay of beta unstable radioactive ions. These unstable ions are created by impinging a target with high energy protons. Subsequently, the ions are collected, bunched, accelerated and stored in a decay ring. The standard design of the decay ring comprises of a racetrack shaped tunnel. When the ions decay along the straight sections, they produce a $\nu_{e}$ or $\bar{\nu}_{e}$ beam. 


\begin{tabular}{|c|c|c|c|c|c|}
\hline Ion & $\tau(\mathrm{s})$ & $E_{0}(\mathrm{MeV})$ & $f$ & Decay fraction & Beam \\
\hline${ }_{10}^{18} \mathrm{Ne}$ & 2.41 & 3.92 & 820.37 & $92.1 \%$ & $\nu_{e}$ \\
${ }_{2}^{6} \mathrm{He}$ & 1.17 & 4.02 & 934.53 & $100 \%$ & $\bar{\nu}_{e}$ \\
\hline${ }_{5}^{8} \mathrm{~B}$ & 1.11 & 14.43 & 600872.07 & $100 \%$ & $\nu_{e}$ \\
${ }_{3}^{8} \mathrm{Li}$ & 1.20 & 13.47 & 425355.16 & $100 \%$ & $\bar{\nu}_{e}$ \\
\hline
\end{tabular}

Table 1: Beta decay parameters: lifetime $\tau$, electron total end-point energy $E_{0}, f$-value and decay fraction for various ions [58].

This neutrino beam would be very suitable for precision experiments because it is mono-flavor and hence beam related backgrounds are almost absent. The neutrino spectrum depends only on the beta decay end-point energy $E_{0}$ and the Lorentz boost of the radioactive ions $\gamma$. The spectral shape can therefore be very well determined. The flux normalization is given directly by $N_{\beta}$, the number of useful ion decays per year in the straight section of the storage ring. The standard numbers taken for the ${ }^{18} \mathrm{Ne}$ and ${ }^{6} \mathrm{He}$ are $1.1 \times 10^{18}\left(\nu_{e}\right)$ and $2.9 \times 10^{18}\left(\bar{\nu}_{e}\right)$ useful decays per year, respectively [57]. Wherever not explicitly mentioned, these reference numbers of useful ion decays for $\nu_{e}$ and $\bar{\nu}_{e}$ are chosen. Note, however, that new ideas suggest luminosities higher even by a factor of ten or so, depending on the isotopes used, by using a recirculating ring to improve the performance of the ion source $[53,54]$. The total luminosity is given by the product of useful ion decays/year $\times$ running time $\times$ detector mass $\times$ detection efficiency. Throughout the study we will consider five years of neutrino and five years of antineutrino running. Since the reference luminosity might not be reachable for different reasons, or it may be much higher because of a better ion source, larger detector, etc., we will include it as a parameter in this study.

The beam divergence is controlled by the Lorentz boost $\gamma$. Hence by increasing $\gamma$, we can produce a higher beam collimation and increase the beam intensity along the forward direction $\propto \gamma^{2}$. However, note that though the intensity can be increased by choosing either a higher $N_{\beta}$ or $\gamma$, they might produce very different neutrino beams. While increasing $N_{\beta}$ merely increases the overall normalization of the flux, increasing $\gamma$ increases both the total flux as well as the average energy of the beam. This dependence of the beam flux on $\gamma$ is illustrated in Fig. 1. Notice that we assume the same $\gamma$ for both neutrino as well as antineutrino modes.

Another crucial aspect associated with Beta-beams is the choice of the beta unstable ion. The properties that a suitable ion should have include a high production yield, large decay fraction, reasonably long lifetime, and preferably lower $Z / A$ ratio. The most widely discussed ions are ${ }^{18} \mathrm{Ne}$ and ${ }^{6} \mathrm{He}$, which would produce a $\nu_{e}$ and $\bar{\nu}_{e}$ beam respectively. The details of the source ions for Beta-beams are given in Table 1 . The other pair of ions which have been proposed as an alternative to ${ }^{18} \mathrm{Ne}$ and ${ }^{6} \mathrm{He}$, are ${ }^{8} \mathrm{~B}$ and ${ }^{8} \mathrm{Li}[53,54]$. The main difference between ${ }^{8} \mathrm{~B}+{ }^{8} \mathrm{Li}$ compared to ${ }^{18} \mathrm{Ne}+{ }^{6} \mathrm{He}$ is the higher end-point energy (see Table 1 ). The factor $\sim 3.68$ (3.35) difference in end-point energy ensures that for the same peak (anti)neutrino energy, approximately given by $\gamma E_{0}$, the $\gamma$ required for ${ }^{8} \mathrm{~B}\left({ }^{8} \mathrm{Li}\right)$ will be 3.68 (3.35) times smaller than that needed for ${ }^{18} \mathrm{Ne}\left({ }^{6} \mathrm{He}\right)$. Since we assume the same $\gamma$ for both ions within each pair, we use the average difference in the endpoint energy $(3.35+3.68) / 2 \simeq 3.5$ to estimate the effects of $\gamma$. The isotope dependence can be also seen in Fig. 1. The figure shows that the peak energy is approximately given by $\gamma E_{0}$. 

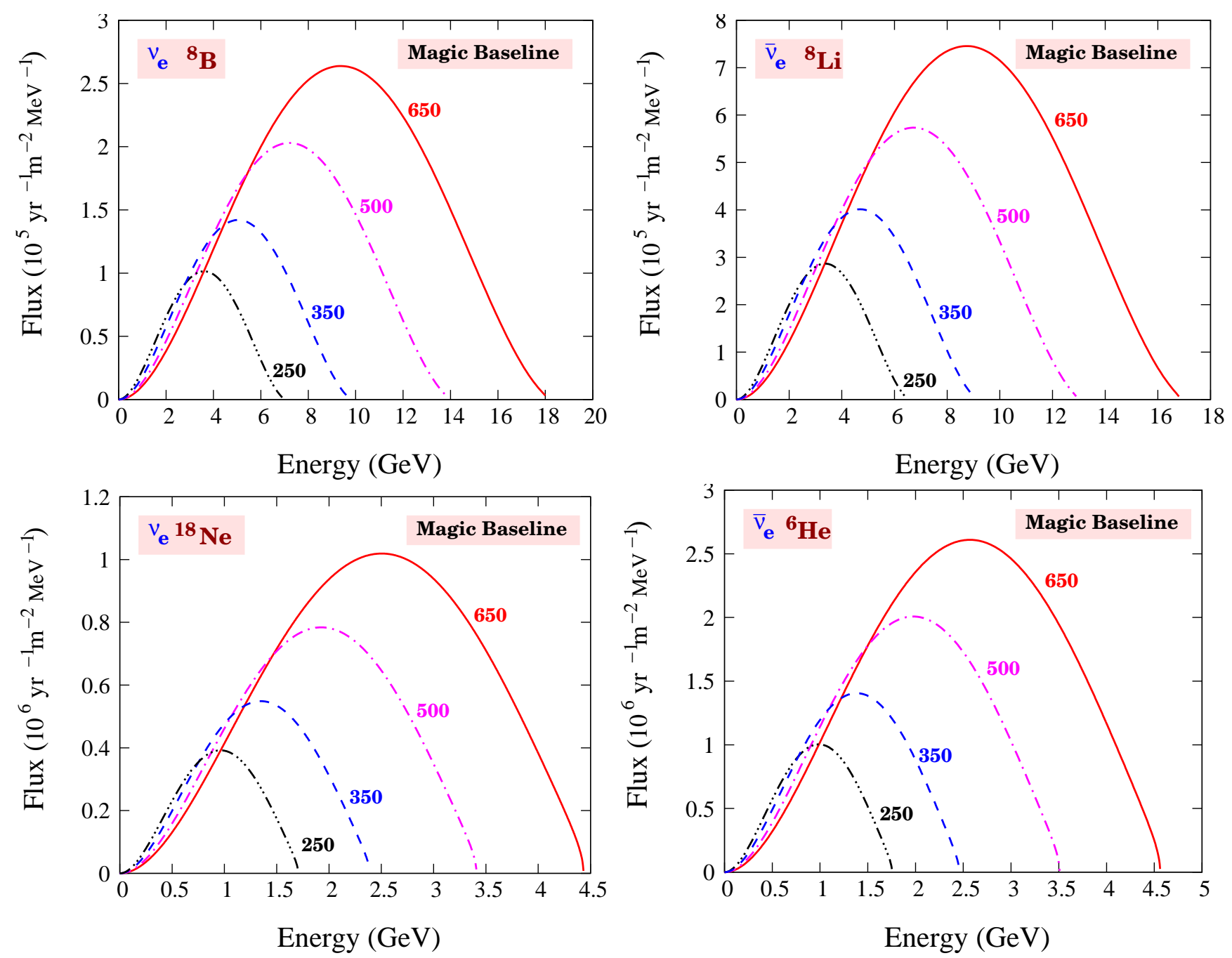

Figure 1: The unoscillated Beta-beam flux spectrum arriving at a detector placed at the magic baseline. The upper panels are for ${ }^{8} \mathrm{~B}$ (left panel) and ${ }^{8} \mathrm{Li}$ (right panel), while the lower panels are for ${ }^{18} \mathrm{Ne}$ (left panel) and ${ }^{6} \mathrm{He}$ (right panel).

Let us discuss now the conditions to obtain a similar spectrum (including normalization) when using different isotope pairs at the same baseline. The purpose of this exercise is to derive the conditions under which we produce matching neutrino energies and fluxes, and therefore deal with the same physics (including neutrino energies, statistics, and, especially, matter effects). If we neglect effects of the endpoint in the beta beam spectrum (i.e., $\left.E_{0} \gg m_{e}\right)$, we know from the beta beam flux formula that the peak energy of the spectrum is approximately given by $\gamma E_{0}$, and the total flux is proportional to $N_{\beta} \gamma^{2}$. In order to obtain a spectrum with the same peak energy and normalization for two different isotopes with very different endpoint energies (such as ${ }^{8} \mathrm{~B}$ and ${ }^{18} \mathrm{Ne}$ ), we therefore have the following conditions (modulo endpoint effects):

$$
\frac{N_{\beta}^{(1)}}{N_{\beta}^{(2)}} \simeq\left(\frac{E_{0}^{(1)}}{E_{0}^{(2)}}\right)^{2}, \quad \frac{\gamma^{(1)}}{\gamma^{(2)}} \simeq \frac{E_{0}^{(2)}}{E_{0}^{(1)}}
$$


From these matching conditions, one obtains the ratio of $N_{\beta}^{(1)} / N_{\beta}^{(2)}$ and $\gamma^{(1)} / \gamma^{(2)}$ needed for the source ions. Therefore, using an isotope with a higher endpoint energy allows for a lower $\gamma$ to obtain the same neutrino energies. However, in order to get the same flux, the useful isotope decays have to be adjusted quadratically. For our pairs of isotopes, we have $E_{0}^{B+L i} \simeq 3.5 \cdot E_{0}^{N e+H e}$. Therefore, from Eq. (1) we obtain the conditions

$$
N_{\beta}^{B+L i} \simeq 12 \cdot N_{\beta}^{N e+H e}, \quad \gamma^{N e+H e} \simeq 3.5 \cdot \gamma^{B+L i}
$$

in order to obtain the same neutrino flux spectrum. Note that the number of useful ion decays $N_{\beta}$ represents, to first approximation, an ion source degree of freedom, whereas the $\gamma$ is an accelerator degree of freedom. ${ }^{4}$ Each can be adjusted with completely different technical challenges. The actual optimization between higher $\gamma$ versus higher isotope production rates depends on individual cost and machine aspects, and cannot be done at this place [59].

The optimal baseline depends crucially on the choice of source ions and the boost factor. For shorter baselines one is away from the matter resonance, and hence the flux arriving at the detector is proportional to $1 / L^{2}$. If one wants to stay at the oscillation maximum in vacuum, one has $L / E=$ const., and therefore $L \propto \gamma$. Since the cross sections are proportional to $\sim E \propto \gamma$ for deep inelastic scattering (DIS) processes, one has an overall $1 / L^{2} \times \gamma \times \gamma^{2}=\gamma$ scaling of the event rates in the DIS regime. Close to matter resonance, the flux at the detector hardly falls as a function of $L$, which means that longer baselines might be preferred. This qualitative discussion of the baseline dependence does not take into account the non-trivial dependence of the oscillation probabilities on the oscillation parameters, and the intrinsic degeneracies. It is the purpose of this work to study this dependence.

From the discussion above we see that one has to optimize for the Beta-beam flux itself by a judicious choice of:

- The types of ions and their end-point energy.

- Lorentz boost factor, $\gamma$.

- Number of useful ion decays per year, $N_{\beta}$.

It is clear that every choice of ion, $\gamma, N_{\beta}$ and $L$ will give a different physics reach for the experiment. The choice of $\gamma$ and $N_{\beta}$ will determine the initial Beta-beam flux for a given choice of the source ions. This is what we would call the "input" of the experiment. What finally determines the physics reach of the experiment is the number of events seen in the detector, and the potential to resolve correlations and degeneracies. We will call this the "output" of the experiment. The aim of course is to maximize the "ouput". However, there are practical limitations on stretching the "input" possibilities. Keeping these in mind, we study the comparitive sensitivity reach of the greenfield Beta-beam set-ups in two ways:

1. By fixing the input and comparing the output.

2. By fixing the output and comparing the required input.

\footnotetext{
${ }^{4}$ There is, however, a non-negligible effect of $N_{\beta}$ on the accelerator by increasing the number of ions per bunch (or the number of bunches).
} 


\begin{tabular}{|l|c|}
\hline Total Mass & $50 \mathrm{kton}$ \\
Energy threshold & $1 \mathrm{GeV}$ \\
Detection Efficiency $(\epsilon)$ & $80 \%$ \\
Charge Identification Efficiency $\left(f_{I D}\right)$ & $95 \%$ \\
Detector Energy Resolution function $(\sigma)$ & $0.15 \mathrm{E}$ \\
Bin Size & $1 \mathrm{GeV}$ \\
NC Background Rejection & 0.0001 \\
Signal error & $2.5 \%$ \\
Background error & $5 \%$ \\
\hline
\end{tabular}

Table 2: Detector characteristics for neutrinos/antinuetrinos used in the simulations. The bin size is kept fixed, while the number of bins is varied according to the maximum energy.

We will use approach (1) in section 3, where we make a comparison between the sensitivity reach of the experiment using either the ${ }^{8} \mathrm{~B}$ and ${ }^{8} \mathrm{Li}$ combination or the ${ }^{18} \mathrm{Ne}$ and ${ }^{6} \mathrm{He}$ combination, as a function of $L$. Sections 4 and 5 are more in the spirit of approach (2).

\subsection{The Detector}

We are interested in measuring the golden channel probability $P_{e \mu}$. Since we have a $\nu_{e}\left(\bar{\nu}_{e}\right)$ flux in the beam, we need a detector which is sensitive to muons (antimuons). The detector should have a suitable energy threshold, depending on the energy spectrum of the Beta-beam. In addition, it should have a good energy resolution and low backgrounds. There are a number of detector technologies that have been considered in the literature. For the low energy Beta-beams, water $\breve{C}$ Cerenkov detectors are the most widely chosen, mainly because of their low energy threshold and large size. This is a very well known and tested detector technology. In addition, the detector can be relatively easily upgraded; typically megaton-sizes [60,61, 62] appear in the literature. However, the backgrounds in this detector are generally larger than in other detector types. For an intermediate $\gamma$ Beta-beam, a Totally Active Scintillator Detector (TASD) is a possible technology. This is the option chosen and studied by the $\mathrm{NO} \nu \mathrm{A}$ collaboration [12]. The third kind of detector technology, which has been studied extensively and which is currently being used by MINOS, is the magnetized iron calorimeter. A larger version is envisaged to come up soon at the INO facility in India [55]. In this paper it will be referred to as ICAL@INO. For both TASD and magnetized iron detectors the background rejection is typically considered to be better than for water Čerenkov detectors.

In this paper we use, for the sake of simplicity, only one type of detector for both types of ions and all values of $\gamma$. We use an ICAL@INO type of detector configuration [55]. We give the details of our detector specifications in Table 2. The charge identification efficiency is incorporated since 
that helps in reducing the neutral current backgrounds. The number of (anti)muon events in the detector is given by

$$
N_{i}=T n_{n} f_{I D} \epsilon \int_{0}^{E_{\max }} d E \int_{E_{A_{i}}^{\min }}^{E_{A_{i}}^{\max }} d E_{A} \phi(E) \sigma_{\nu_{\mu}}(E) R\left(E, E_{A}\right) P_{e \mu}(E),
$$

where $T$ is the total running time (taken as five years), $n_{n}$ are the number of target nucleons in the detector, $f_{I D}$ is the charge identification efficiency and $R\left(E, E_{A}\right)$ is the energy resolution function of the detector, for which we assume a Gaussian function. For muon (antimuon) events, $\sigma_{\nu_{\mu}}$ is

the neutrino (antineutrino) interaction cross-section. The quantities $E$ and $E_{A}$ are the true and reconstructed (anti)neutrino energy respectively.

\subsection{Neutrino Propagation and Simulation Details}

The expression for $P_{e \mu}$ in matter $[63,64,65]$, up to second order terms in the small quantities $\theta_{13}$ and $\alpha \equiv \Delta m_{21}^{2} / \Delta m_{31}^{2}$, is given by $[8,66]$

$$
\begin{aligned}
P_{e \mu} & \simeq \sin ^{2} \theta_{23} \sin ^{2} 2 \theta_{13} \frac{\sin ^{2}[(1-\hat{A}) \Delta]}{(1-\hat{A})^{2}} \\
& \pm \alpha \sin 2 \theta_{13} \sin 2 \theta_{12} \sin 2 \theta_{23} \sin \delta_{\mathrm{CP}} \sin (\Delta) \frac{\sin (\hat{A} \Delta)}{\hat{A}} \frac{\sin [(1-\hat{A}) \Delta]}{(1-\hat{A})} \\
& +\alpha \sin 2 \theta_{13} \sin 2 \theta_{12} \sin 2 \theta_{23} \cos \delta_{\mathrm{CP}} \cos (\Delta) \frac{\sin (\hat{A} \Delta)}{\hat{A}} \frac{\sin [(1-\hat{A}) \Delta]}{(1-\hat{A})} \\
& +\alpha^{2} \cos ^{2} \theta_{23} \sin ^{2} 2 \theta_{12} \frac{\sin ^{2}(\hat{A} \Delta)}{\hat{A}^{2}},
\end{aligned}
$$

where

$$
\Delta \equiv \frac{\Delta m_{31}^{2} L}{4 E}, \quad \hat{A} \equiv \frac{A}{\Delta m_{31}^{2}},
$$

and $A= \pm 2 \sqrt{2} G_{F} N_{e} E$ is the matter potential, given in terms of the electron density $N_{e}$ and (anti)neutrino energy $E$; the plus sign refers to neutrinos while the minus to antineutrinos. The second term in Eq. (4) is CP violating. While we will use this formula to discuss our results in some cases, our simulation is based on the exact probabilities.

Unless stated otherwise, we have generated our simulated data for the benchmark values in the first column of Table 3. These values have been chosen in conformity with the status of the oscillation parameters in the light of the current neutrino data [7]. The values of $\sin ^{2} 2 \theta_{13}$ (true), $\delta_{\mathrm{CP}}$ (true) and mass hierarchy which are allowed to vary in our study, will be mentioned wherever applicable. For the Earth matter density, we use the PREM profile [67]. We expect to have a better knowledge of all the parameters mentioned in Table 3 when the Beta-beam facility comes up. In particular, we assume that the $1 \sigma$ error on them will be reduced to the values shown in the second column of Table $3[14,68,69]$. Therefore, we impose "priors" on these quantities, with the corresponding $1 \sigma$ error. The results presented in section 3 have been generated using the $\chi^{2}$ technique and numerical code described in [40,41]. Figures in sections 4 and 5 have been 


\begin{tabular}{|l||l|}
\hline $\mid \Delta m_{31}^{2}$ (true $) \mid=2.5 \times 10^{-3} \mathrm{eV}^{2}$ & $\sigma\left(\Delta m_{31}^{2}\right)=1.5 \%$ \\
$\sin ^{2} 2 \theta_{23}($ true $) \mid=1.0$ & $\sigma\left(\sin ^{2} 2 \theta_{23}\right)=1 \%$ \\
$\Delta m_{21}^{2}($ true $)=8.0 \times 10^{-5} \mathrm{eV}^{2}$ & $\sigma\left(\Delta m_{21}^{2}\right)=2 \%$ \\
$\sin ^{2} \theta_{12}($ true $)=0.31$ & $\sigma\left(\sin ^{2} \theta_{12}\right)=6 \%$ \\
$\rho($ true $)=1($ PREM $)$ & $\sigma(\rho)=5 \%$ \\
\hline
\end{tabular}

Table 3: Chosen benchmark values of oscillation parameters and their $1 \sigma$ estimated errors. The last row gives the corresponding values for the Earth matter density.

generated using the GLoBES package [70]. For the latter simulations we do not put any priors on $\left|\Delta m_{31}^{2}\right|$ and $\sin ^{2} 2 \theta_{13}$ and instead add 10 year prospective disappearance data from T2K [28]. All other details of the $\chi^{2}$ technique, as well as beam and detector specification, are taken identical in both numerical codes. We have made extensive checks, and the results obtained from both codes match to a reasonably high level of accuracy. This robustness of the results can be regarded as an independent cross-check within our study.

\section{Optimizing the Baseline}

We optimize the Beta-beam experiments separately with respect to the following physics outputs:

1. The $\theta_{13}$ measurement reach.

2. The mass hierarchy reach.

3. The CP sensitivity reach.

By "reach" we refer to going to as small $\sin ^{2} 2 \theta_{13}$ as possible. We define the performance indicators below, and optimize the Beta-beam experiment with respect to the baseline in this section. Note that we fix the $\gamma$ as well as the number of useful ion decays to their reference values in this section.

\subsection{The $\theta_{13}$ Sensitivity/Discovery Reach}

We define two sets of performance indicators for quantifying the sensitivity of the experiment to $\theta_{13}$. We call them the " $\theta_{13}$ sensitivity reach" and the " $\theta_{13}$ discovery reach". The $\theta_{13}$ sensitivity reach is defined as the range of $\sin ^{2} 2 \theta_{13}$ which is incompatible with the data generated for $\sin ^{2} 2 \theta_{13}$ (true) $=$ 0 at the $3 \sigma$ CL. This performance indicator corresponds to the new $\sin ^{2} 2 \theta_{13}$ limit if the experiment does not see a signal for $\theta_{13}$-driven oscillations ${ }^{5}$. In that case, we can exclude some allowed values

\footnotetext{
${ }^{5}$ Note from Eq. (4), while the first three terms go to zero when $\theta_{13} \rightarrow 0$, the last term, which depends only on the solar parameters and $\theta_{23}$, remains non-vanishing. Therefore, when the flux is high, i.e., for large $\gamma$ and/or enhanced luminosity, we expect a sizable number of events even when $\sin ^{2} 2 \theta_{13}$ (true) $=0$.
} 


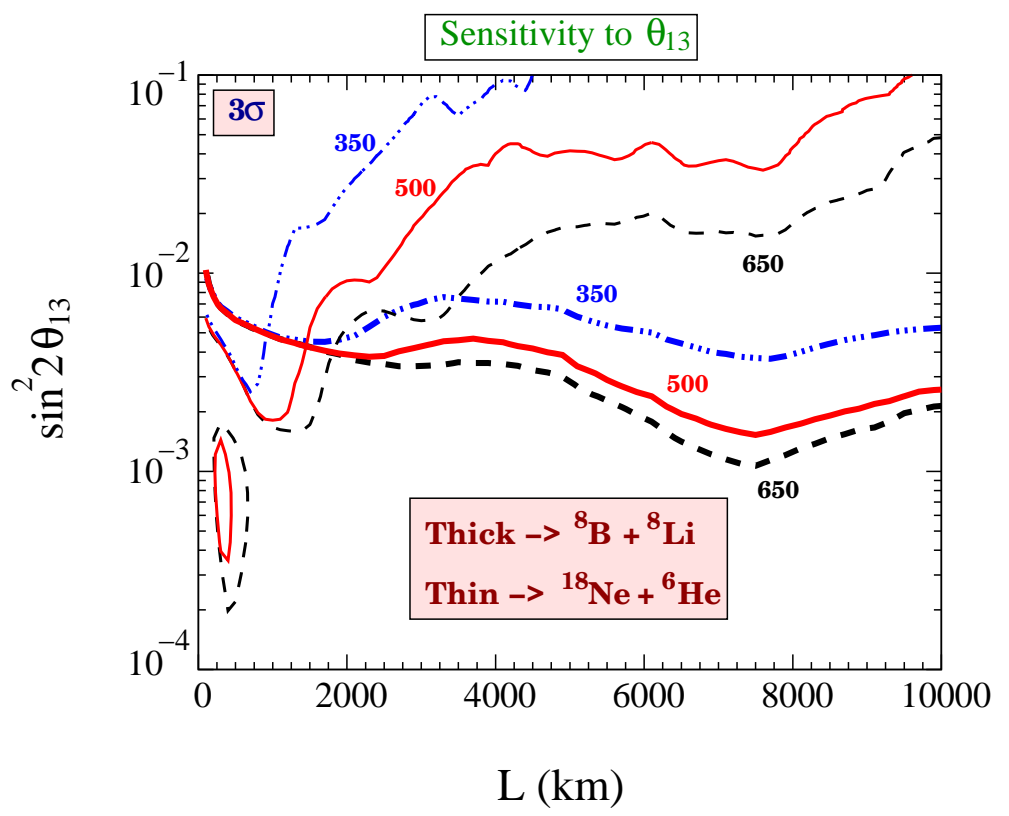

Figure 2: The $\theta_{13}$ sensitivity reach as a function of the baseline for three different values of $\gamma=350$ (blue dot-dashed lines), 500 (red solid lines) and 650 (black dashed lines). The thick lines show the results for ${ }^{8} \mathrm{~B}$ and ${ }^{8} \mathrm{Li}$ as source ions, while the thin lines give the corresponding results for ${ }^{18} \mathrm{Ne}$ and ${ }^{6} \mathrm{He}$. The region above the curves/within the isolated islands are permitted by the sensitivity criterion.

of $\sin ^{2} 2 \theta_{13}$, which we call our " $\theta_{13}$ sensitivity reach". In Fig. 2 , we show the $L$-dependence of the $\theta_{13}$ sensitivity reach of the Beta-beam experiment. The thick lines show the sensitivity for the ${ }^{8} \mathrm{~B}$ and ${ }^{8} \mathrm{Li}$ combination, while the thin lines show the corresponding sensitivities for the ${ }^{18} \mathrm{Ne}$ and ${ }^{6} \mathrm{He}$ ions. The results are shown for three different values of $\gamma$. Since the true value of $\theta_{13}$ is assumed to be zero, the data is independent of the true neutrino mass hierarchy and $\delta_{\mathrm{CP}}$ (true), but the fit depends on $\operatorname{sgn}\left(\Delta \mathrm{m}_{31}^{2}\right)$ and $\delta_{\mathrm{CP}}$. We have marginalized our results over all oscillation parameters, including mass hierarchy and $\delta_{\mathrm{CP}}$. We have also marginalized over the normalization of the Earth matter density ${ }^{6}$. For the ${ }^{8} \mathrm{~B}$ and ${ }^{8} \mathrm{Li}$ combination, the best $\theta_{13}$ sensitivity is obtained at the magic baseline. This baseline is defined by the condition [35]

$$
\sin (\hat{A} \Delta) \simeq 0,
$$

which evaluates to $\sqrt{2} G_{F} n_{e} L\left(n_{e}\right)=2 \pi$, or $L \simeq 7000$ to $7500 \mathrm{~km}$. Therefore, the second, third and last terms in Eq. (4) vanish at this baseline. Since the second and third terms are the CPdependent terms (with the second term being CP violating), the effect of $\delta_{\mathrm{CP}}$ is absent. Therefore, the correlation and degeneracies are hardly present, increasing the sensitivity of the experiment.

The impact of the magic baseline is particularly visible for the ${ }^{8} \mathrm{~B}$ and ${ }^{8} \mathrm{Li}$ combination because for these ions the fluxes peak at $E \sim 5-10 \mathrm{GeV}$ for $\gamma \sim 350-650$. It turns out that for these

\footnotetext{
${ }^{6}$ In all results given in this paper, we have done full marginalization over hierarchy, all oscillation parameters and the normalization factor of the Earth matter density distribution.
} 

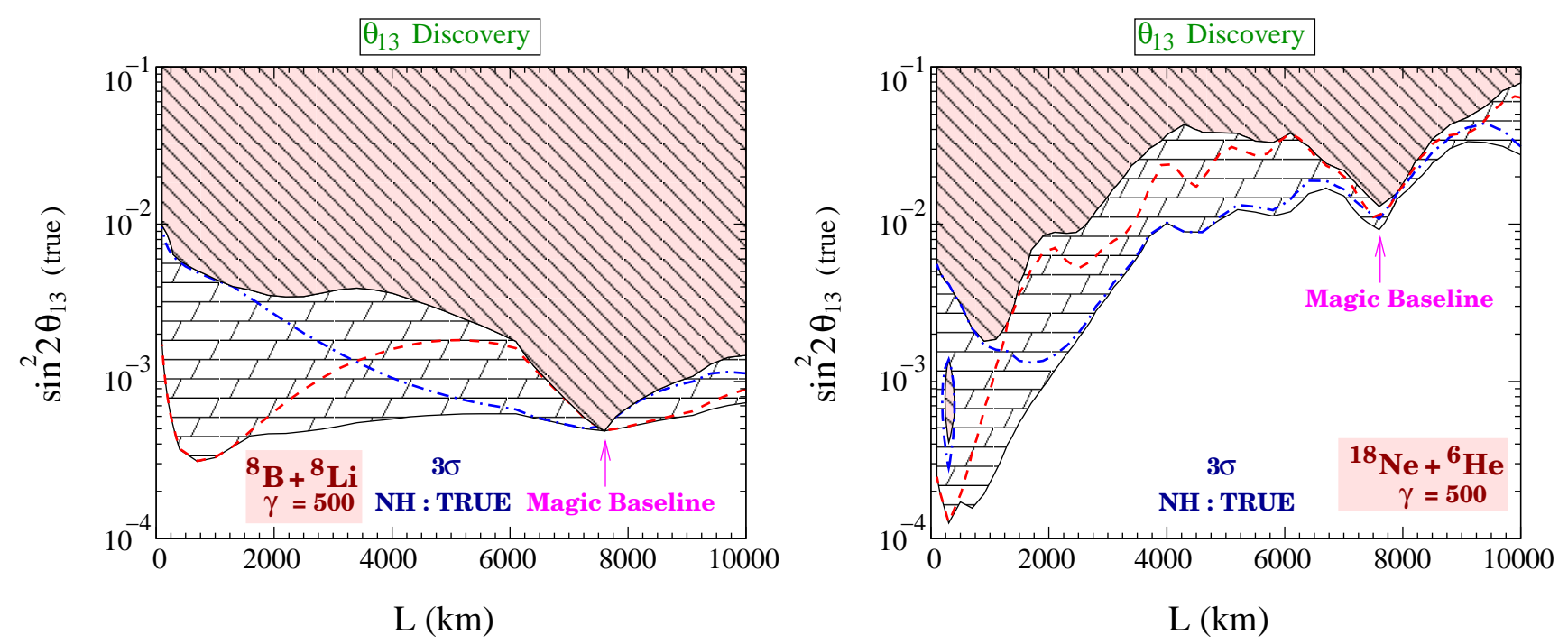

Figure 3: The $\theta_{13}$ discovery reach as a function of baseline for ${ }^{8} \mathrm{~B}$ and ${ }^{8} \mathrm{Li}$ (left panel) and for ${ }^{18} \mathrm{Ne}$ and ${ }^{6} \mathrm{He}$ (right panel). The pink hatched region is where a $3 \sigma$ discovery is possible for all values of $\delta_{\mathrm{CP}}$ (true), the white boxed region is where a $3 \sigma$ discovery is possible for some values of $\delta_{\mathrm{CP}}$ (true), while the unshaded blank region is where its impossible to get a discovery at $3 \sigma$ for any value of $\delta_{\mathrm{CP}}$ (true). The red dashed curve is the discovery reach for $\delta_{\mathrm{CP}}$ (true) $=0$ while the blue dashed-dotted curve is the same for $\delta_{\mathrm{CP}}$ (true) $=\pi$. Here the true hierarchy is assumed to be normal.

energies, one obtains near-resonant matter effects, corresponding to $\hat{A} \rightarrow 1$ in Eq. (4). Therefore, the flux decreases less than $1 / L^{2}$ at these energies, and is still quite substantial at the magic baseline. In fact, for short distances, the $1 / L^{2}$ dependence is canceled by the resonant probability enhancement, as can be read off Eq. (4). For the ${ }^{18} \mathrm{Ne}$ and ${ }^{6} \mathrm{He}$ combination, the fluxes peak around $E \sim 1.0-2.5 \mathrm{GeV}$ for $\gamma \sim 350-650$. For such low energies, matter effects are small, even for very long baselines. More importantly, for $E \sim 1.0-2.5 \mathrm{GeV}$, the oscillatory factor peaks at $L \sim 500-1250 \mathrm{~km}$ if one assumes $\Delta m_{31}^{2}=2.5 \times 10^{-3} \mathrm{eV}^{2}$. Therefore, for this ion pair, the minimum in the $\sin ^{2} 2 \theta_{13}$ sensitivity comes at the baseline where we expect the first oscillation maximum. For $\gamma=650,500$ and 350 , the best sensitivity comes at $L=1250 \mathrm{~km}, 890 \mathrm{~km}$ and $680 \mathrm{~km}$, respectively. Beyond this baseline, both the flux and the probability fall, resulting in a sharp loss of the $\sin ^{2} 2 \theta_{13}$ sensitivity. Note the isolated regions for ${ }^{18} \mathrm{Ne}$ and ${ }^{6} \mathrm{He}$ and $\gamma=500$ and 650 , which are also incompatible with $\sin ^{2} 2 \theta_{13}$ (true) $=0$. The gap is mainly an artifact of the presence of clone solutions at these smaller baselines and it might be breached by the combination of a higher flux, better energy resolution, etc., leading to a much better sensitivity.

In Fig. 3, we show the "discovery reach" for $\sin ^{2} 2 \theta_{13}$ (true). This performance indicator is defined as the range of $\sin ^{2} 2 \theta_{13}$ (true) values which allow us to rule out $\sin ^{2} 2 \theta_{13}=0$ at the $3 \sigma$ CL. Since the data are now generated for a non-zero $\sin ^{2} 2 \theta_{13}$ (true), there is a $\delta_{\mathrm{CP}}$ (true) dependence and a true mass hierarchy dependence. The discovery reach for $\delta_{\mathrm{CP}}$ (true) $=0$ is shown by the dashed curve in Fig. 3 , and the discovery reach for $\delta_{\mathrm{CP}}$ (true) $=\pi$ is shown by the dashed-dotted curve. For each $\delta_{\mathrm{CP}}$ (true), one obtains a corresponding such curve. To show the impact of this $\delta_{\mathrm{CP}}$ (true) dependence of the discovery potential, and to illustrate explicitly the 
increase in the "risk factor" coming from our lack of knowledge of $\delta_{\mathrm{CP}}$ (true), we present in Fig. 3 a band marked by boxes showing the entire range of $\sin ^{2} 2 \theta_{13}$ (true) values corresponding to all possible values of $\delta_{\mathrm{CP}}$ (true). Fig. 3 has been drawn for the true normal hierarchy.

The way to interpret this figure is as follows: At a given $L$, one will discover $\sin ^{2} 2 \theta_{13}$ for any $\delta_{\mathrm{CP}}$ (true) at the upper limit aand beyond (the pink hatched region), whereas there is no value of $\delta_{\mathrm{CP}}$ (true) for which one can discover $\sin ^{2} 2 \theta_{13}$ below the lower limit (the unshaded region). Within the band, the fraction of $\delta_{\mathrm{CP}}$ (true), which allows for a discovery, increases as one approaches the upper limit. Therefore, the upper edge of this band gives the most conservative discovery reach. We will take this as our final discovery reach at a given baseline. The lower edge of the band shows the best possible case. The left panel of Fig. 3 is computed for ${ }^{8} \mathrm{~B}$ and ${ }^{8} \mathrm{Li}$, while the right panel is computed for ${ }^{18} \mathrm{Ne}$ and ${ }^{6} \mathrm{He}$. All results in this figure have been computed for $\gamma=500$. Again we note that, at the magic baseline, our results are CP independent, and the band reduces to a point since the discovery reach is independent of $\delta_{\mathrm{CP}}$ (true) at this baseline ${ }^{7}$. For the ${ }^{8} \mathrm{~B}$ and ${ }^{8} \mathrm{Li}$ combination the best discovery reach comes at $L=7600$, which is the magic baseline. It is noteworthy though that for these ions the best possible case, given by the lower edge of the band, comes at a lower baseline of $L \simeq 700 \mathrm{~km}$. For the ${ }^{18} \mathrm{Ne}$ and ${ }^{6} \mathrm{He}$ pair, since matter effects are low, there is only a local minimum at the magic baseline. In fact, the best performance of the experiment comes at $L=900 \mathrm{~km}$, which is approximately the baseline where we have the oscillation maximum for $\gamma=500$. Again we note the appearance of islands inside the band for the ${ }^{18} \mathrm{Ne}$ and ${ }^{6} \mathrm{He}$ ions. For regions inside these islands, the discovery of $\sin ^{2} 2 \theta_{13}$ is independent of $\delta_{\mathrm{CP}}$ (true).

We have also computed the $\sin ^{2} 2 \theta_{13}$ (true) discovery reach for the true inverted hierarchy. Since the figures look very similar to Fig. 3, we do not show them explicitely. However, note that in the inverted case, the curves for $\delta_{\mathrm{CP}}$ (true) $=0$ and $\pi$ interchange their roles. It is not surprising that the inverted hierarchy performs similar to the normal one for the beta beams, since the neutrino and antineutrino event rates are very similar. Our chosen number of useful ion decay is about a factor of three higher for antineutrinos than neutrinos, which is compensated by the higher neutrino cross sections.

\subsection{The $\operatorname{sgn}\left(\Delta \mathrm{m}_{31}^{2}\right)$ Sensitivity Reach}

We define the mass hierarchy sensitivity as the range of $\sin ^{2} 2 \theta_{13}$ (true) for which the wrong hierarchy can be excluded at the $3 \sigma \mathrm{CL}$. We show our results as a function of the baseline in Fig. 4. As before, the left panel is for the ${ }^{8} \mathrm{~B}$ and ${ }^{8} \mathrm{Li}$ case, and the right panel for ${ }^{18} \mathrm{Ne}$ and ${ }^{6} \mathrm{He}$. In addition, we show the risk with respect to $\delta_{\mathrm{CP}}$ (true) as a band marked by boxes, where the lower edge corresponds to the best possible reach (obtainable for only some specific $\delta_{\mathrm{CP}}(\operatorname{true})$ ), and the upper edge to the conservative case (valid irrespective of the value of $\delta_{\mathrm{CP}}(\operatorname{true})$ ). That means that the hierarchy can be determined for any $\sin ^{2} 2 \theta_{13}$ (true) above the upper end of the band. In all panels, we also show the curves corresponding to $\delta_{\mathrm{CP}}$ (true) $=0$ and $\delta_{\mathrm{CP}}($ true $)=\pi$, for illustration. For the ${ }^{8} \mathrm{~B}$ and ${ }^{8} \mathrm{Li}$ case, we find that the best sensitivity to the mass hierarchy comes at the magic baseline. The reason for this is basically the same as in the previous subsection:

\footnotetext{
${ }^{7}$ For the ${ }^{18} \mathrm{Ne}$ and ${ }^{6} \mathrm{He}$ combination, there is a small width even at the magic baseline. In this case $\sin ^{2} 2 \theta_{13}($ true) is large, and Eq. (4) has to be expanded to higher order. The magic baseline condition Eq. (6) may not hold for higher order terms. This was also noted and pointed out in [41].
} 

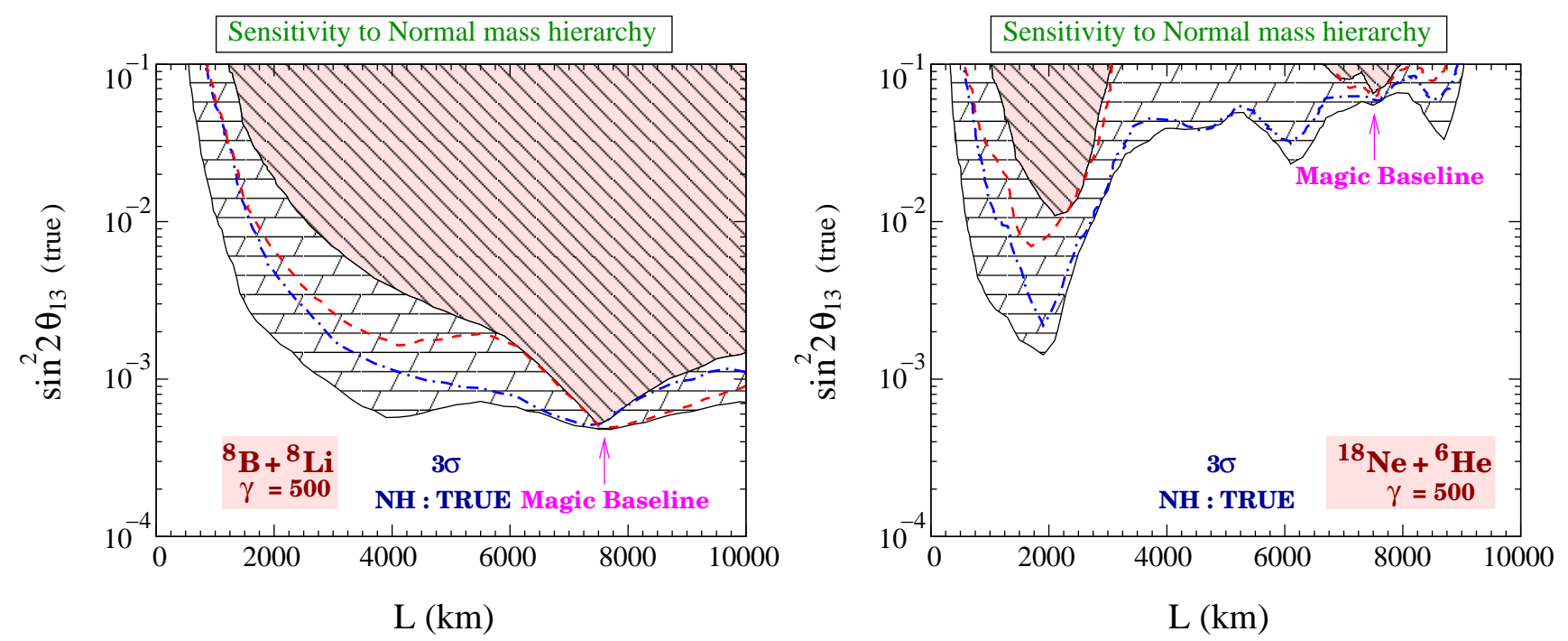

Figure 4: $\sin ^{2} 2 \theta_{13}$ (true) reach for determining $\operatorname{sgn}\left(\Delta \mathrm{m}_{31}^{2}\right)$ at $3 \sigma$ CL as a function of the baseline with ${ }^{8} \mathrm{~B}$ and ${ }^{8} \mathrm{Li}$ (left panel) and ${ }^{18} \mathrm{Ne}$ and ${ }^{6} \mathrm{He}$ (right panel) taken as the source ions. The pink hatched region is where the wrong hierarchy can be ruled out for all values of $\delta_{\mathrm{CP}}$ (true), the white boxed region is where the hierarchy determination is possible for some values of $\delta_{\mathrm{CP}}$ (true), while the unshaded blank region is where its impossible to determine the hierarchy for any value of $\delta_{\mathrm{CP}}$ (true). The red dashed (blue dashed-dotted) curves show the hierarchy sensitivity for $\delta_{\mathrm{CP}}($ true $)=0\left(\delta_{\mathrm{CP}}(\right.$ true $\left.)=\pi\right)$. The true hierarchy is assumed to be normal.

The near-resonant matter effects lead to a large number of events, and the resonant behavior is only present for one hierarchy (normal or inverted). It is, therefore, possible to have very large matter dependent oscillations for $L \gtrsim 4000 \mathrm{~km}$. However, the effect of $\delta_{\mathrm{CP}}$ (true) could wash away the sensitivity to $\operatorname{sgn}\left(\Delta \mathrm{m}_{31}^{2}\right)$. For example, for $L \simeq 4000 \mathrm{~km}$, we do not obtain a very good sensitivity for even the best case. If one takes into account all possible $\delta_{\mathrm{CP}}$ (true) values, the sensitivity becomes deteriorated significantly. At the magic baseline, the dependence on $\delta_{\mathrm{CP}}$ (true) is reduced. Therefore, this baselines provides the best choice to determine the mass hierarchy.

The right panel in Fig. 4 corresponds to the ${ }^{18} \mathrm{Ne}$ and ${ }^{6} \mathrm{He}$ case. The sensitivity to the mass hierarchy is rather poor for the values of $\gamma$ we have adopted here because of the low energies off the matter resonance (the energies are about a factor of 3.5 lower than for the ${ }^{8} \mathrm{~B}$ and ${ }^{8} \mathrm{Li}$ pair). Since the mass hierarchy determination crucially depends on matter effects, we have very poor sensitivity for this performance indicator, at least for the values of $\gamma$ considered here. We will see in the next section that this set of ions could start giving comparable sensitivity only when the $\gamma$ is increased by a factor of three. For $\gamma=500$, the best sensitivity comes at $L \simeq 2000 \mathrm{~km}$.

Again, we have tested the true inverted hierarchy case, and we have not found any significant qualitative or quantitative differences.

\subsection{The CP Sensitivity Reach}

We next discuss the reach of the experiment to $\mathrm{CP}$ violation as a function of $L$. We define the sensitivity to (maximal) $\mathrm{CP}$ violation as the range of $\sin ^{2} 2 \theta_{13}$ for which $\mathrm{CP}$ conservation (i.e., 

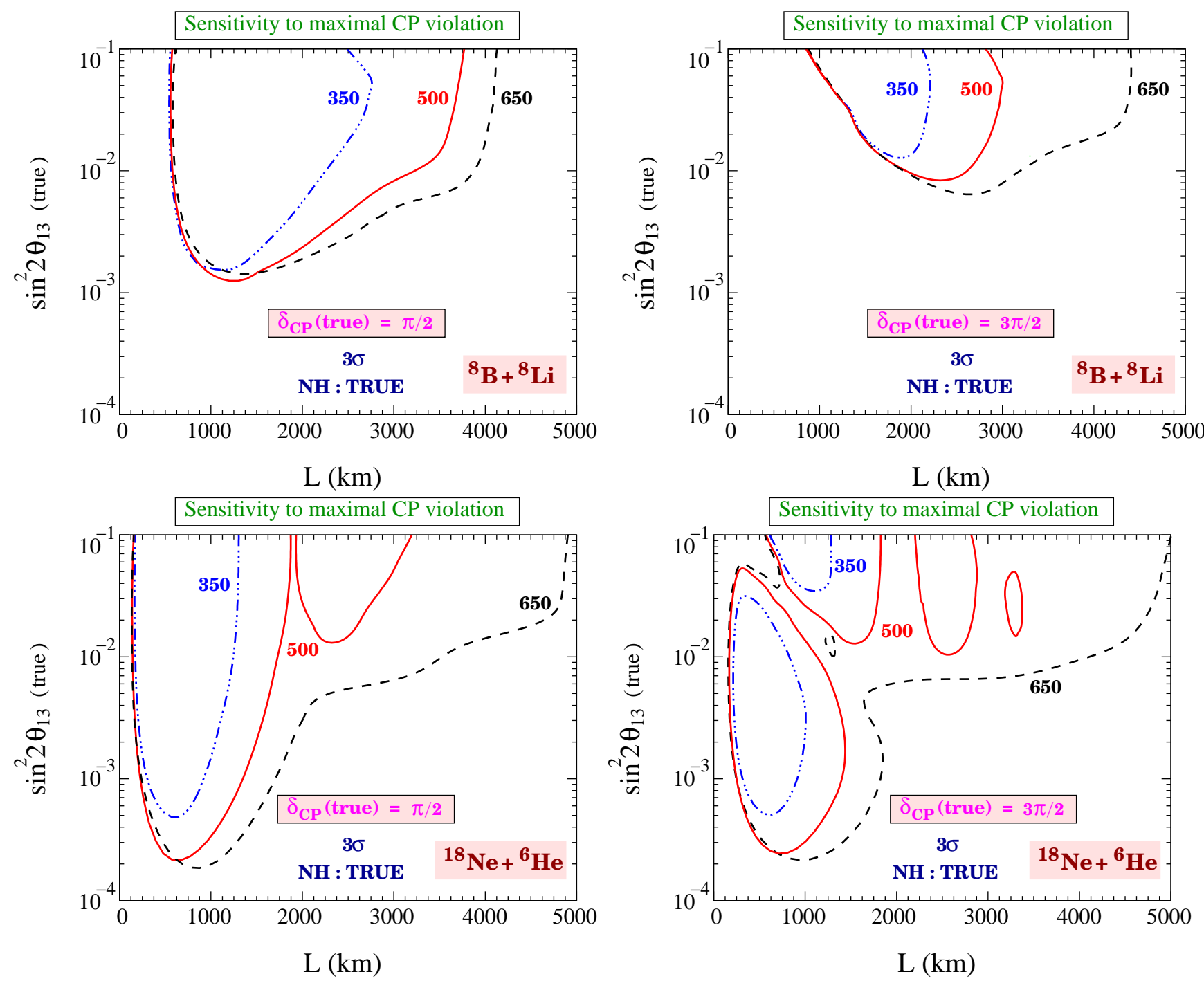

Figure 5: $\sin ^{2} 2 \theta_{13}$ (true) reach for sensitivity to maximal $\mathrm{CP}$ violation as a function of $L$, for three different values of $\gamma$. The upper panels are for ${ }^{8} \mathrm{~B}$ and ${ }^{8} \mathrm{Li}$ taken as the source ions and the lower panels are for ${ }^{18} \mathrm{Ne}$ and ${ }^{6} \mathrm{He}$ as the source ions. The left panels are for $\delta_{\mathrm{CP}}$ (true) $=\pi / 2$, and the right panels for $\delta_{\mathrm{CP}}$ (true) $=3 \pi / 2$. In all cases, a true normal hierarchy has been assumed. 
$\delta_{\mathrm{CP}}=0$ and $\pi$ ) can be excluded at the $3 \sigma$ confidence level irrespective of the (fit) hierarchy. The results are shown in Fig. 5, where we generate the data either for $\delta_{\mathrm{CP}}$ (true) $=\pi / 2$ (left panels) or $3 \pi / 2$ (right panels). The upper panels are computed for the ${ }^{8} \mathrm{~B}$ and ${ }^{8} \mathrm{Li}$ pair, while the lower panels are for the ${ }^{18} \mathrm{Ne}$ and ${ }^{6} \mathrm{He}$ pair. We show results for three choices of $\gamma$. Let us focus on $\delta_{\mathrm{CP}}($ true $)=\pi / 2$ (left panels) first. The best choice for the baseline is around $L=500-1500$ $\mathrm{km}$, depending on the choice of ions and $\gamma$. For example, for the ${ }^{8} \mathrm{~B}$ and ${ }^{8} \mathrm{Li}$ combination, the optimum is found at around the FNAL-Homestake baseline $L=1290 \mathrm{~km}$. However, the absolute performance for ${ }^{8} \mathrm{~B}$ and ${ }^{8} \mathrm{Li}$ is worse than for the ${ }^{18} \mathrm{Ne}$ and ${ }^{6} \mathrm{He}$ combination. For $3 \pi / 2$ (right panels), the sensitivity becomes worse in both cases due to the impact of degeneracies. Ignoring the gaps, there is still a substantial CP violation reach for ${ }^{18} \mathrm{Ne}$ and ${ }^{6} \mathrm{He}$ for both $\delta_{\mathrm{CP}}$ (true) $=\pi / 2$ and $3 \pi / 2$. For example, if Double Chooz constrains $\sin ^{2} 2 \theta_{13}$ to values smaller than about 0.04 , a short baseline $L \simeq 300 \mathrm{~km}$ together with $\gamma \geq 500$ might be sufficient for the $\mathrm{CP}$ violation measurement because the gap can be excluded. The optimal baseline for maximum $\sin ^{2} 2 \theta_{13}$ reach, on the other hand, ranges between $L \simeq 600$ for $\gamma=350$ to $L=1000 \mathrm{~km}$ for $\gamma=650$. For the inverted hierarchy, we obtain very similar figures to Fig. 5 , but the role of $\delta_{\mathrm{CP}}$ (true) $=\pi / 2$ and $\delta_{\mathrm{CP}}$ (true) $=3 \pi / 2$ is exchanged.

\section{Optimizing the Baseline and Gamma}

In this section, we will allow for larger values of $\gamma$ and show results in the $\gamma-L$ plane, while we still fix the number of useful ion decays to its reference value. In section 2 , we have compared the different ion pairs for the same fixed $\gamma$. This however, implies that the peak energies, and therefore the physics (matter effects etc.) is different. Here we wish to show the projected sensitivity for the two sets of isotopes when they produce beams with similar peak energies, where we leave both $L$ and $\gamma$ as free parameters. The motivation is to have similar effect of the oscillations and matter effects for both the sets of ions. The end-point energies of ${ }^{18} \mathrm{Ne}$ and ${ }^{6} \mathrm{He}$ are about 3.5 times smaller than those of ${ }^{8} \mathrm{~B}$ and ${ }^{8} \mathrm{Li}$. Therefore, with $\gamma$ for ${ }^{18} \mathrm{Ne}$ and ${ }^{6} \mathrm{He}$ scaled up by a factor of roughly 3 , we expect almost the same oscillated spectral shape for the two cases. Hence we show the results for $\gamma<1000$ for ${ }^{8} \mathrm{~B}$ and ${ }^{8} \mathrm{Li}$, and for $\gamma<3000$ for the ${ }^{18} \mathrm{Ne}$ and ${ }^{6} \mathrm{He}$. We stress that we allow for these prohibitively large values of $\gamma$ for ${ }^{18} \mathrm{Ne}$ and ${ }^{6} \mathrm{He}$ in order to compare set-ups with same physics output. While they might appear to be unrealistic, they can in principle be achieved by an accelerator as large as the LHC [71]. The Tevatron might also be used to give large boost factors.

\subsection{The $\theta_{13}$ Sensitivity}

In order to discuss the $\theta_{13}$ performance, we use the $\sin ^{2} 2 \theta_{13}$ sensitivity reach in this section. The $\sin ^{2} 2 \theta_{13}$ sensitivity represents the largest fit $\sin ^{2} 2 \theta_{13}$ which fits a $\sin ^{2} 2 \theta_{13}$ (true) $=0$. Therefore in this section, we do not take into account any disjoint regions, such as the ones which appeared in Fig. 2. We reiterate that the $\sin ^{2} 2 \theta_{13}$ sensitivity is independent of the true mass hierarchy and $\delta_{\mathrm{CP}}$ (true). We show in Fig. 6 the $3 \sigma \sin ^{2} 2 \theta_{13}$ sensitivity reach as a function of baseline $L$ and Lorentz factor $\gamma$. The curves in this figure represent the $3 \sigma$ C.L. contours for different values of $\sin ^{2} 2 \theta_{13}$. The contours are spaced by 0.2 in $\log _{10}\left(\sin ^{2} 2 \theta_{13}\right)$, and the numbers are shown in the figure for some of them. The diamonds mark the absolute optimum within each plot; the 

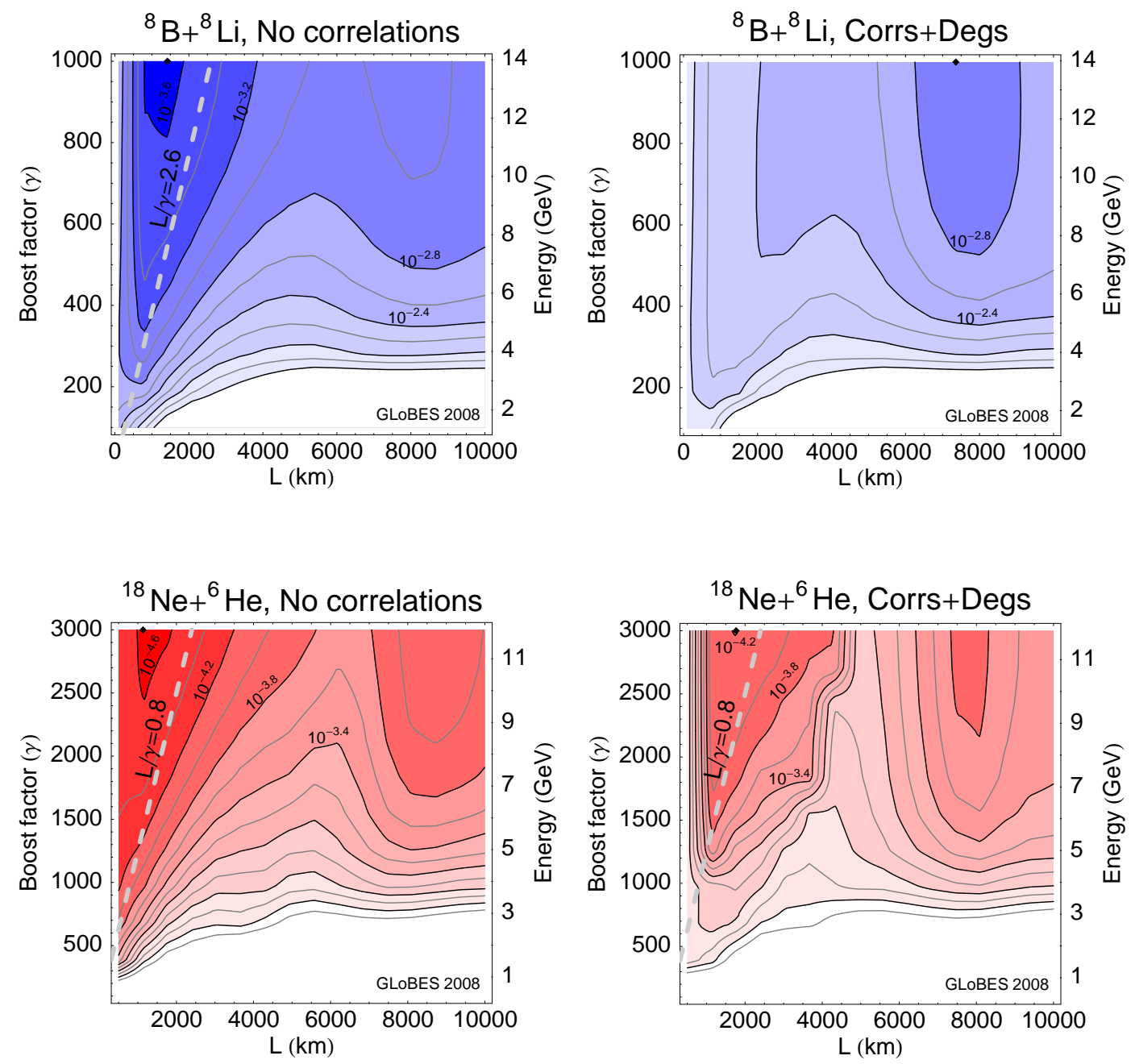

Figure 6: The $3 \sigma \sin ^{2} 2 \theta_{13}$ sensitivity as a function of baseline $L$ and boost factor $\gamma$. The $\sin ^{2} 2 \theta_{13}$ sensitivity represents the largest possible $\sin ^{2} 2 \theta_{13}$, which fits data simulated at $\sin ^{2} 2 \theta_{13}$ (true) $=0$. The upper row corresponds to ${ }^{8} \mathrm{~B}$ and ${ }^{8} \mathrm{Li}$, the lower row to ${ }^{18} \mathrm{Ne}$ and ${ }^{6} \mathrm{He}$. The left column shows the systematics limit only (i.e., the oscillation parameters are fixed with $\delta_{\mathrm{CP}}=0$ ), whereas the right column shows the final limit including correlations and degeneracies. The contours are spaced by 0.2 in $\log _{10}\left(\sin ^{2} 2 \theta_{13}\right)$, where the numbers are given for some of these. The diamonds mark the absolute optimum within each plot, which are $10^{-3.66}, 10^{-3.00}, 10^{-4.66}$, and $10^{-4.21}$, respectively, from the upper left to the lower right. On the right axes of the plots, an energy scale is attached which corresponds to the mean peak energy $\gamma \bar{E}_{0}$ with $\bar{E}_{0}$ being the mean of the endpoint energies for the isotope pair. 
corresponding values are given in the figure caption. The upper row corresponds to ${ }^{8} \mathrm{~B}$ and ${ }^{8} \mathrm{Li}$ and the lower row to ${ }^{18} \mathrm{Ne}$ and ${ }^{6} \mathrm{He}$. The left column shows the systematics limit only, where we have kept all parameters including the mass hierarchy and $\delta_{\mathrm{CP}}=0$ fixed in the fit. The right column shows the final sensitivity limit after including correlations and degeneracies. From Section 2.1, we have learned that a gamma range for ${ }^{18} \mathrm{Ne}$ and ${ }^{6} \mathrm{He}$ about three times larger than for ${ }^{8} \mathrm{~B}$ and ${ }^{8} \mathrm{Li}$ gives similar neutrino peak energies, which is illustrated by the right vertical axes in the plots. This feature is reflected in our choice of the $\gamma$ ranges in Fig. 6. Note that in both the upper and lower rows of Fig. 6 we have used the same isotope decay rates, the same running times, and the same detector simulation.

Let us first focus on the systematics limit shown in the left column. The two panels for the different isotopes look qualitatively very similar, but the absolute performance is much better for ${ }^{18} \mathrm{Ne}$ and ${ }^{6} \mathrm{He}$ for $\gamma$ values three times larger. This is because while we have tuned the oscillation probability for both isotopes to be the same, the flux increases as $\gamma^{2}$, and therefore the event rate for the ${ }^{18} \mathrm{Ne}$ and ${ }^{6} \mathrm{He}$ combination scales up by a factor of about nine for the three times higher boost factors. According to Eq. (2), the absolute sensitivities for the two isotopes would be more or less identical if the $\gamma$ for ${ }^{18} \mathrm{Ne}$ and ${ }^{6} \mathrm{He}$ was turned up by a factor of about 3.5 and the source luminosity for ${ }^{8} \mathrm{~B}$ and ${ }^{8} \mathrm{Li}$ was enhanced by a factor of about 12 . This means that primary difference between the upper and lower rows in Fig. 6 is the different event rate, but not the spectral shape and energies. We can also note from the figure that for a give set of source ions and for a given $L$ and $N_{\beta}$, the sensitivity of the experiment increases as $\gamma$ is increased. For the best sensitivity reach, the baseline has then to be adjusted accordingly. The figure shows that the best choice for $L$ roughly corresponds to tuning $L / \gamma \simeq 2.6$ for ${ }^{8} \mathrm{~B}$ and ${ }^{8} \mathrm{Li}$ and $L / \gamma \simeq 0.8$ for ${ }^{18} \mathrm{Ne}$ and ${ }^{6} \mathrm{He}^{8}$ We show the lines for these conditions in Fig. 6 in the upper left panel and lower panels ${ }^{9}$. Note that the slopes of these lines are different by about a factor of $E_{0}^{(1)} / E_{0}^{(2)} \simeq 3.5$ because the boost factors are related by Eq. (2) in order to obtain the same neutrino energies. The optimal regions appear slightly to the left of these lines in the figure because statistics are higher at lower $L$.

Once the correlations and degeneracies are included (c.f., right column of Fig. 6), the optimum baseline changes qualitatively for ${ }^{8} \mathrm{~B}$ and ${ }^{8} \mathrm{Li}$, but not for ${ }^{18} \mathrm{Ne}$ and ${ }^{6} \mathrm{He}$. One can read off from the upper right panel that the magic baseline becomes the optimum baseline for about $\gamma \geq 350$, whereas for $\gamma<350$ the shorter baseline is preferred. The reason for this was also discussed in the previous section. For $\gamma \geq 350$ one gets a peak neutrino energy greater than $5 \mathrm{GeV}$. For these energies one can obtain very large matter effects for the very long baselines and hence the oscillation probability becomes larger here. Therefore, the event rate in this regime is improved due to a combination of large matter driven oscillation probability as well as increased flux driven by larger $\gamma$. Most importantly, close to the magic baseline, the effect of $\delta_{\mathrm{CP}}$ is almost negligible. Therefore, once the correlations and degeneracies are taken into account, this becomes the deciding factor which ensures that for $\gamma \geq 350$, the magic baseline emerges as the most optimal baseline choice for the $\theta_{13}$ sensitivity. For $\gamma<350$, both the oscillation probability as well as the flux collimation are small, and therefore the longer baselines suffer due to the $1 / L^{2}$ suppression factor for the event rates. As a result, despite being free from problems of correlations and degeneracies, the magic

\footnotetext{
${ }^{8}$ In fact, the unit of $L / \gamma$ is $\mathrm{km}$, which we do not put explicitly.

${ }^{9}$ Since for ${ }^{8} \mathrm{~B}$ and ${ }^{8} \mathrm{Li}$ we do not get the optimum at the oscillation maxima obeying $L / \gamma \simeq 2.6$ once correlations and degeneracies are taken, we do not show the line in the upper right panel.
} 
baseline looses sensitivity compared to the shorter baselines. Also note that the sensitivity for $\gamma<350$ is rather poor, and therefore it is desirable to do the experiment at $\gamma \geq 350$.

The situation is very different for the ${ }^{18} \mathrm{Ne}$ and ${ }^{6} \mathrm{He}$ case, where the shorter baseline (which roughly satisfies $L / \gamma \simeq 0.8$ ) is always the better choice for the $\sin ^{2} 2 \theta_{13}$ sensitivity for any value of $\gamma$. Note that we have adjusted $\gamma$ to keep the same peak energy (shown by the dashed lines) and hence we have the same spectral shape and the same effect of the oscillation probability as compared to ${ }^{8} \mathrm{~B}$ and ${ }^{8} \mathrm{Li}$. However, the $\gamma$ here is about three times larger, and that becomes the overwhelming deciding factor for the most suitable baseline. The enhancement in the flux due to the beam collimation in this case ensures that the shorter baselines have a high enough statistics to handle the problem of parameter correlations and degeneracies. Therefore, the $\sin ^{2} 2 \theta_{13}$ sensitivity is better than that of the magic baseline, which is free from a $\delta_{\mathrm{CP}}$ dependence but suffers from the $1 / L^{2}$ suppression. Therefore, the best sensitivity roughly follows the $L / \gamma=0.8$ line. We remind the reader that the isolated regions obtained for $\gamma=500$ and 650 in Fig. 2 also correspond to $L / \gamma \simeq 0.8$. For small values of $\sin ^{2} 2 \theta_{13}$, that are relevant here, the $\delta_{\mathrm{CP}}$ dependent terms in the fit are extremely important at values of $L$ close to $L_{\text {oscmax }} / 2$. This corresponds to $L / \gamma \simeq 0.8$ for ${ }^{18} \mathrm{Ne}$ and ${ }^{6} \mathrm{He}$.

In the following section, we will compare the long (magic) with the short baseline. For the long (magic) baseline, we will choose $L=7500 \mathrm{~km}$ (optimum). For the short baseline we will take the local optimum obtained, which obeys $L / \gamma \simeq 2.6$ for ${ }^{8} \mathrm{~B}$ and ${ }^{8} \mathrm{Li}$ and $L / \gamma \simeq 0.8$ for ${ }^{18} \mathrm{Ne}$ and ${ }^{6} \mathrm{He}$.

\subsection{The $\operatorname{sgn}\left(\Delta \mathrm{m}_{31}^{2}\right)$ Sensitivity Reach}

In Fig. 7 , we show the $\sin ^{2} 2 \theta_{13}$ (true) reach for the sensitivity to the normal mass hierarchy $(3 \sigma)$ as a function of baseline $L$ and boost factor $\gamma$. The $\operatorname{sgn}\left(\Delta \mathrm{m}_{31}^{2}\right)$ reach represents the minimum $\sin ^{2} 2 \theta_{13}$ (true) above which the mass hierarchy will be discovered for any $\sin ^{2} 2 \theta_{13}$ (true) (i.e., there are no gaps in the sensitivity). The upper row corresponds to ${ }^{8} \mathrm{~B}$ and ${ }^{8} \mathrm{Li}$, the lower row to ${ }^{18} \mathrm{Ne}$ and ${ }^{6} \mathrm{He}$. The left column is computed for $\delta_{\mathrm{CP}}$ (true) $=\pi / 2$, whereas the right column is for $\delta_{\mathrm{CP}}$ (true) $=3 \pi / 2$. Note that the $\sin \delta$ term in Eq. (4) is positive for neutrinos and negative for antineutrinos. This means that the simulated neutrino rate is larger for $\delta_{\mathrm{CP}}($ true $)=\pi / 2$ and smaller for $\delta_{\mathrm{CP}}($ true $)=3 \pi / 2$. Therefore, statistics is better for $\delta_{\mathrm{CP}}$ (true) $=\pi / 2$, and we expect a better mass hierarchy sensitivity. It is for this reason we have chosen to illustrate the $\operatorname{sgn}\left(\Delta \mathrm{m}_{31}^{2}\right)$ sensitivity reach for $\delta_{\mathrm{CP}}$ (true) $=\pi / 2$ and $3 \pi / 2$ only in Fig. 7 , which represent two cases close to the best case and worst case. These two cases would change their role if one used a true inverted hierarchy instead, i.e., the best performance would be close to $\delta_{\mathrm{CP}}$ (true) $=3 \pi / 2$, and the worst close to $\delta_{\mathrm{CP}}($ true $)=\pi / 2$.

For both isotope pairs, there are two main observations for the mass hierarchy measurement:

1. Longer baselines are preferred with the optimal sensitivity reach appearing at $L$ close to the magic baseline. This is not surprising since for longer baselines the matter effect contribution becomes larger, which allows to discriminate between $\mathrm{NH}$ and $\mathrm{IH}$. The sensitivity gets better at the magic baseline since the probability at the magic baseline is free of $\delta_{\mathrm{CP}}$ related correlations and degeneracies.

2. Higher boost factors are preferred (at least within the shown ranges). First of all, the event 

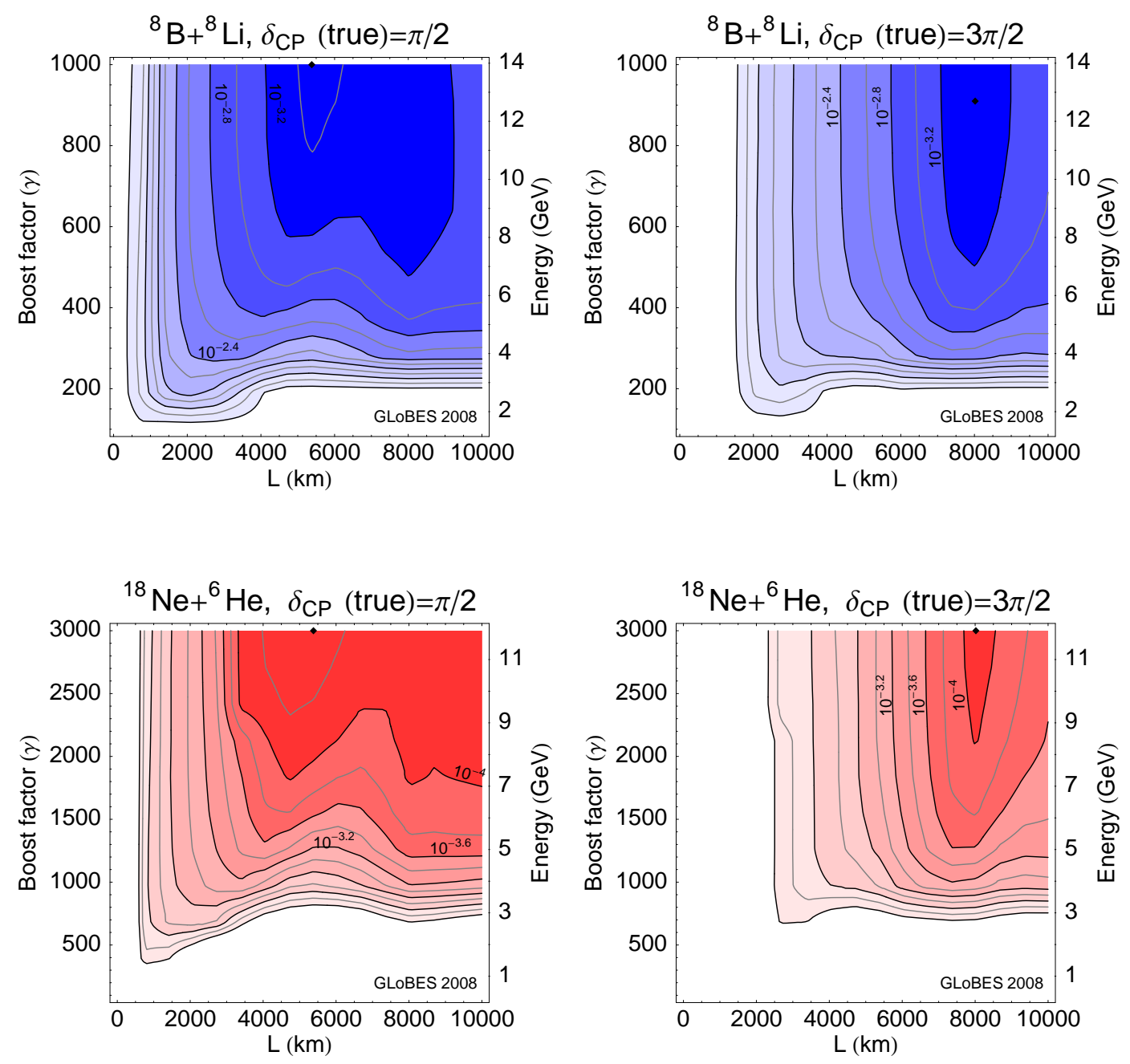

Figure 7: The $\sin ^{2} 2 \theta_{13}$ (true) reach for the sensitivity to the normal mass hierarchy $(3 \sigma)$ as a function of the baseline $L$ and boost factor $\gamma$. The $\sin ^{2} 2 \theta_{13}$ (true) reach represents the minimum $\sin ^{2} 2 \theta_{13}$ (true) above which the mass hierarchy will be discovered for any $\sin ^{2} 2 \theta_{13}$ (true) (i.e., there are no gaps in the sensitivity). The upper row corresponds to ${ }^{8} \mathrm{~B}$ and ${ }^{8} \mathrm{Li}$, while the lower row to ${ }^{18} \mathrm{Ne}$ and ${ }^{6} \mathrm{He}$. The left column is computed for $\delta_{\mathrm{CP}}$ (true) $=\pi / 2$, whereas the right column is for $\delta_{\mathrm{CP}}($ true $)=3 \pi / 2$. The contours are spaced by 0.2 in $\log _{10}\left(\sin ^{2} 2 \theta_{13}\right.$ (true)), where the numbers are given for some of these. The diamonds mark the absolute optimum within each plot, which are $10^{-3.46}, 10^{-3.36}, 10^{-4.29}$, and $10^{-4.10}$, respectively, from the upper left to the lower right. On the right axes of the plots, an energy scale is attached which corresponds to the mean peak energy $\gamma \bar{E}_{0}$ with $\bar{E}_{0}$ being the mean of the endpoint energies for the isotope pair. 
rate increases as $\gamma$ increases. However, matter effects also increase as one approaches the mantle resonance energy at about $7 \mathrm{GeV}$. Therefore, sufficiently high energies are needed to observe the mass hierarchy discriminating matter effects.

In summary, we find that the optimal choice for determining the hierarchy is $L \sim 7000-$ $9000 \mathrm{~km}$, and $\gamma \gg 200$ for ${ }^{8} \mathrm{~B}$ and ${ }^{8} \mathrm{Li}$ or $\gamma \gg 750$ for ${ }^{18} \mathrm{Ne}$ and ${ }^{6} \mathrm{He}$. Of course, for the same energy, the absolute performance is better for ${ }^{18} \mathrm{Ne}$ and ${ }^{6} \mathrm{He}$ as long as one can create boost factors which are three times larger.

\subsection{The CP Sensitivity Reach}

In order to discuss the CP sensitivity, we show in Fig. 8 the $\sin ^{2} 2 \theta_{13}$ (true) reach for the sensitivity to maximal $\mathrm{CP}$ violation $(3 \sigma)$ as a function of the baseline $L$ and boost factor $\gamma$. The data are generated for $\delta_{\mathrm{CP}}$ (true) $=\pi / 2$ (left panels) or $3 \pi / 2$ (right panels). We show in the figures the minimum $\sin ^{2} 2 \theta_{13}$ (true) above which maximal CP violation will be discovered for any $\sin ^{2} 2 \theta_{13}$ (true). In these figures we discard the lower allowed islands in Fig. 5 (lower right panel) and consider only the upper regions. The upper row corresponds to ${ }^{8} \mathrm{~B}$ and ${ }^{8} \mathrm{Li}$, the lower row to ${ }^{18} \mathrm{Ne}$ and ${ }^{6} \mathrm{He}$. Obviously, for the CP violation sensitivity, a shorter baseline is a must, because the magic baseline is not sensitive to $\delta_{\mathrm{CP}}$. Hence, we only show baselines up to $5000 \mathrm{~km}$ in this figure, since there is no sensitivity for longer baselines. As for the mass hierarchy reach, here too the performance for the normal hierarchy is best for $\delta_{\mathrm{CP}}$ (true) $=\pi / 2$ and worst for $\delta_{\mathrm{CP}}$ (true) $=3 \pi / 2$. However, their roles change for the inverted hierarchy. Note that the poor sensitivity close to $\delta_{\mathrm{CP}}$ (true) $\simeq 3 \pi / 2$ mainly comes from unresolved degeneracies due to poor statistics. Combining data of this set-up with a second (much longer) baseline could help in resolving the degeneracies and improving the sensitivity. Here we focus on the left column of Fig. 8 for the following discussion.

A comparison of the upper left and lower left panels of Fig. 8 reveals qualitative differences

between the sensitivities coming from the two pairs of isotopes. We observe that the ${ }^{18} \mathrm{Ne}$ and ${ }^{6} \mathrm{He}$ combination is far superior for probing the CP phase. We had already seen this feature in the earlier section when we had compared the CP sensitivity reach of the two sets of isotopes for the same $\gamma$. In fact, the sensitivity to maximal $\mathrm{CP}$ violation improves with $\gamma$ for ${ }^{18} \mathrm{Ne}$ and ${ }^{6} \mathrm{He}$, and we find that the best case shown by the diamond appears for the highest $\gamma$ we have taken. For ${ }^{8} \mathrm{~B}$ and ${ }^{8} \mathrm{Li}$ the sensitivity in general is comparatively poorer and does not scale with $\gamma$. In fact, the $\gamma$ dependence of the sensitivity is rather weak with the best CP sensitivity coming for $\gamma \simeq 650$. As far as the possible baselines are concerned, for $\delta_{\mathrm{CP}}$ (true) $=\pi / 2$ the optimal baselines roughly follow the $L / \gamma=2.6$ line for ${ }^{8} \mathrm{~B}$ and ${ }^{8} \mathrm{Li}$, and the $L / \gamma=0.8$ line for ${ }^{18} \mathrm{Ne}$ and ${ }^{6} \mathrm{He}$.

\section{The Impact of Luminosity}

In this section, we study the impact of increasing the overall number of events through either increasing the size of the detector, the exposure time, detector efficiency, or the number of useful ion decays. In order to discuss this, we introduce a luminosity scaling factor multiplying the overall luminosity (useful isotope decays $\times$ running time $\times$ detector mass $\times$ detector efficiency) for both neutrinos and antineutrinos. Note that the luminosity scaling factor corresponds to a reference luminosity, i.e., $1.1 \times 10^{18}\left(\right.$ year $\left.^{-1}\right) \times 5($ year $) \times 50($ kton $) \times 0.76$ for the neutrino beam. 

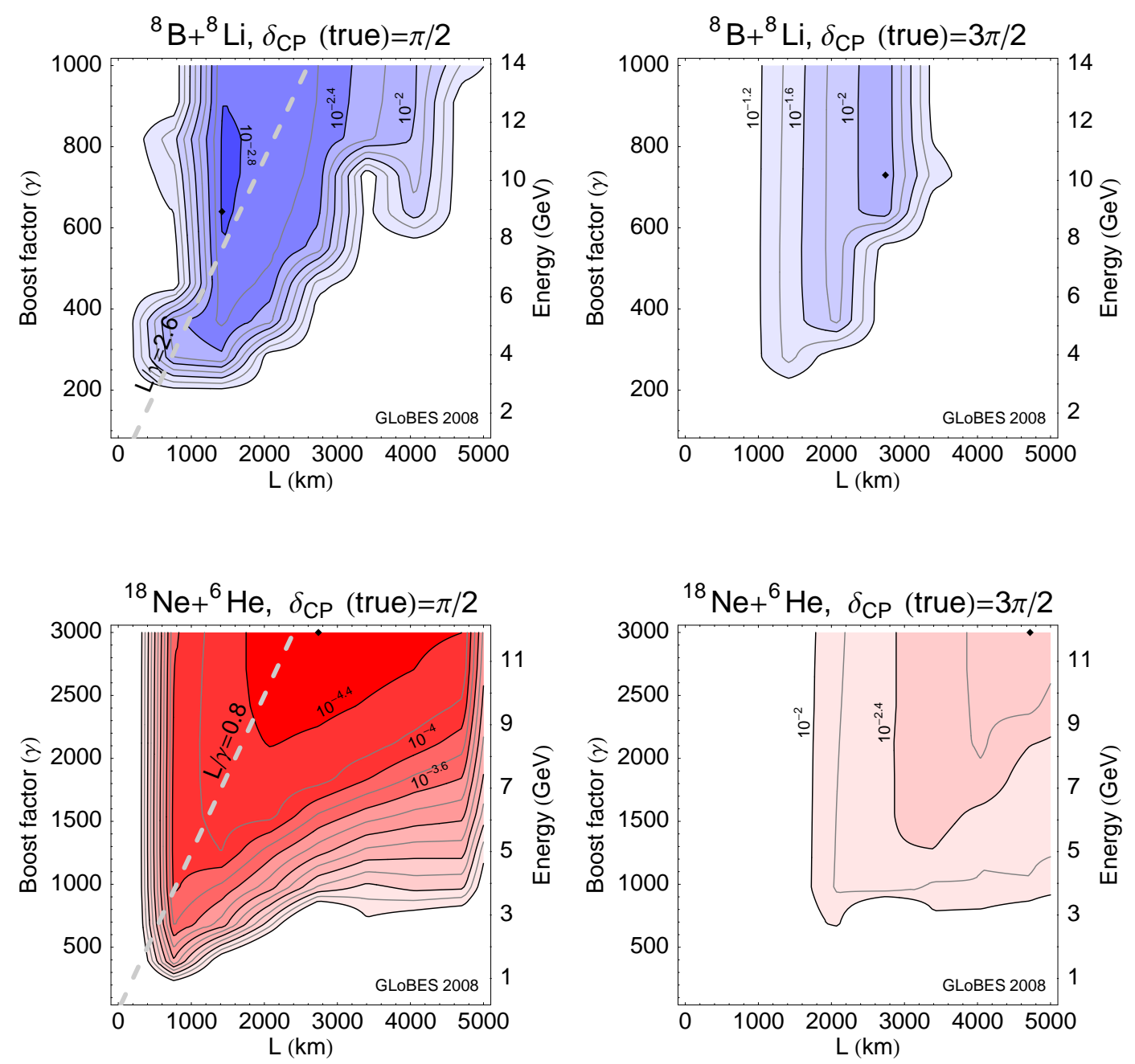

Figure 8: The $\sin ^{2} 2 \theta_{13}$ (true) reach for the sensitivity to maximal CP violation $(3 \sigma)$ as a function of the baseline $L$ and boost factor $\gamma$. The $\sin ^{2} 2 \theta_{13}$ reach represents the minimum $\sin ^{2} 2 \theta_{13}$ (true) above which maximal $\mathrm{CP}$ violation will be discovered for any $\sin ^{2} 2 \theta_{13}$ (true) (i.e., there are no gaps in the sensitivity). The upper row corresponds to ${ }^{8} \mathrm{~B}$ and ${ }^{8} \mathrm{Li}$, the lower row to ${ }^{18} \mathrm{Ne}$ and ${ }^{6} \mathrm{He}$. The left column is computed for $\delta_{\mathrm{CP}}$ (true) $=\pi / 2$, whereas the right column is for $\delta_{\mathrm{CP}}$ (true) $=3 \pi / 2$. The contours are spaced by 0.2 in $\log _{10}\left(\sin ^{2} 2 \theta_{13}\right.$ (true)), where the numbers are given for some of these. The diamonds mark the absolute optimum within each plot. Here a true normal hierarchy is assumed. The diamonds mark the absolute optimum within each plot, which are $10^{-2.82}, 10^{-2.14}$, $10^{-4.50}$, and $10^{-2.79}$, respectively, from the upper left to the lower right. On the right axes of the plots, an energy scale is attached which corresponds to the mean peak energy $\gamma \bar{E}_{0}$ with $\bar{E}_{0}$ being the mean of the endpoint energies for the isotope pair. 
For the antineutrino beam we use $2.9 \times 10^{18}$ useful decays per year. We attempt to determine the optimal value for the luminosity, $\gamma$ and $L$. We fix $\gamma$ at certain benchmark values and compare the performance of short baselines given by $L / \gamma \simeq 2.6$ for ${ }^{8} \mathrm{~B}$ and ${ }^{8} \mathrm{Li}$ and $L / \gamma \simeq 0.8$ for ${ }^{18} \mathrm{Ne}$ and ${ }^{6} \mathrm{He}$, with the magic baseline. For comparison between the isotopes, we use both the approaches discussed in section 2.1. That is, we compare the physics reach of ${ }^{8} \mathrm{~B}$ and ${ }^{8} \mathrm{Li}$ with that of ${ }^{18} \mathrm{Ne}$ and ${ }^{6} \mathrm{He}$, both at same fixed values of $\gamma$ corresponding to the same input, as well as with $\gamma$ for ${ }^{18} \mathrm{Ne}$ and ${ }^{6} \mathrm{He}$ scaled by a factor of about 3.5 to get the same peak neutrino energy, and hence the same neutrino energies. We use the same definition of the performance indicators as in Section 4, i.e., we demand that there is sensitivity for all $\sin ^{2} 2 \theta_{13}$ larger than the given sensitivity limits (which excludes the regions separated by the gaps).

\subsection{The $\theta_{13}$ Sensitivity}

In Fig. 9, we show the $\sin ^{2} 2 \theta_{13}$ sensitivity $(3 \sigma)$ as a function of the luminosity scaling factor. The panels represent the different isotopes and different $\gamma$ values as given in the captions. The dotdashed curves correspond to the magic baseline (MB) with $L=7500 \mathrm{~km}$ fixed, the solid curves to a short baseline with an $L / \gamma$ depending on the isotope. The upper row is for ${ }^{8} \mathrm{~B}$ and ${ }^{8} \mathrm{Li}$. The middle and lower rows are for ${ }^{18} \mathrm{Ne}$ and ${ }^{6} \mathrm{He}$, with the middle row for same $\gamma$ as ${ }^{8} \mathrm{~B}$ and ${ }^{8} \mathrm{Li}$, whereas the lower row is for $\gamma$ 's scaled up by a factor of about 3.5. That means that the middle row represents the same accelerator effort as the upper row in terms of $\gamma$, and the lower row represents similar neutrino energies to the upper row.

There are a number of interesting observations from Fig. 9. First, for the shorter baseline, statistics are crucial for resolving the degeneracies, and we obtain a sudden enhancement of the sensitivity at some value of the luminosity scaling factor. This point is visible as the edge in the luminosity scaling, where the degenerate solution is ruled out at the $3 \sigma$ C.L. For the magic baseline, degeneracies are hardly relevant and we find that the sensitivities exhibit an almost power law scaling with statistics. Second, the curves for the shorter baseline cross the ones for the magic baseline twice in almost all the panels in the upper and lower rows. For ${ }^{8} \mathrm{~B}$ and ${ }^{8} \mathrm{Li}$, the standard assumed luminosity (luminosity scaling factor one) is typically in the window where the magic baseline performs better. Only for the $\gamma=350$ case, the shorter baseline is comparable with the magic baseline for the standard luminosity. For ${ }^{18} \mathrm{Ne}$ and ${ }^{6} \mathrm{He}$, the short baseline is typically better for the standard luminosity and smaller $\gamma$ (middle row). However, for ${ }^{18} \mathrm{Ne}+{ }^{6} \mathrm{He}$ and $\gamma \gg 1000$, already a factor of two loss in luminosity makes the magic baseline the better choice.

Let us now come back to the conditions in Eq. (1). Since we have chosen the upper and lower rows in Fig. 9 such that the gammas scale inverse to the endpoint energies, this formula indicated that the same physics should be obtained for about a factor of 12 difference in luminosity. Let us pick a simple feature where we could test this conclusion: take a look at the edge of the sensitivity jumps for the short baselines. In the lower row, this sensitivity jumps happen for about one order of magnitude less luminosity than in the upper row, which confirms the expectation. This means that a factor of 12 in the number of useful ion decays is indeed needed to reproduce the same physics if one uses ${ }^{8} \mathrm{~B}$ and ${ }^{8} \mathrm{Li}$ instead of ${ }^{18} \mathrm{Ne}$ and ${ }^{6} \mathrm{He}$. However, this comes for a price: A factor of 3.5 higher gamma is needed for ${ }^{18} \mathrm{Ne}+{ }^{6} \mathrm{He}$ than for ${ }^{8} \mathrm{~B}+{ }^{8} \mathrm{Li}$. 

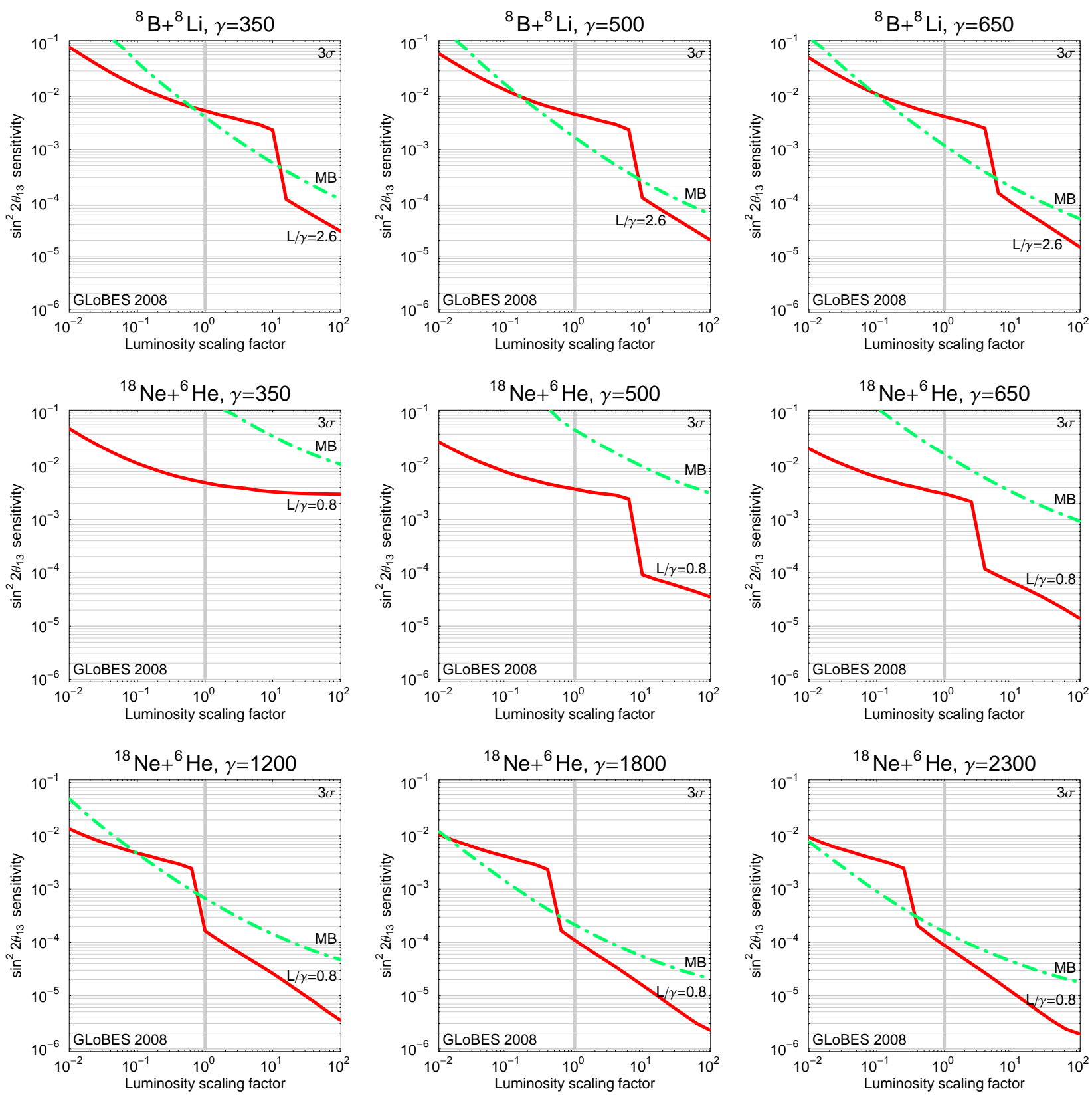

Figure 9: The $\sin ^{2} 2 \theta_{13}$ sensitivity $(3 \sigma)$ as a function of a luminosity scaling factor. The luminosity scaling factor multiplies the overall luminosities (useful isotope decays $\times$ running time $\times$ detector mass $\times$ detector efficiency) for both neutrinos and antineutrinos. The panels represent the different isotopes and different $\gamma$ as indicated in the captions. The green dashed-dotted curves correspond to the magic baseline "MB" with $L=7500 \mathrm{~km}$ fixed, the red solid curves to a short baseline with an $L / \gamma$ depending on the isotope (c.f., lines for fixed $L / \gamma$ in Fig. 6). A true normal hierarchy is assumed. 


\subsection{The $\operatorname{sgn}\left(\Delta \mathrm{m}_{31}^{2}\right)$ Sensitivity Reach}

In Fig. 10, we show the $\sin ^{2} 2 \theta_{13}$ reach for the sensitivity to the normal mass hierarchy $(3 \sigma)$ as a function of the luminosity scaling factor. The panels represent the different isotopes and different $\gamma$ values as given in the captions. The dashed-dotted curves correspond to the long magic baseline "MB" with $L=7500 \mathrm{~km}$ fixed and very little dependence on the true $\delta_{\mathrm{CP}}$, the other two sets of curves to a short baseline with an $L / \gamma$ depending on the isotope (c.f., lines for fixed $L / \gamma$ in Fig. 6). The solid curves are computed for a $\delta_{\mathrm{CP}}$ (true) $=\pi / 2$, the dashed curves for a $\delta_{\mathrm{CP}}($ true $)=3 \pi / 2$.

For the mass hierarchy sensitivity, the magic baseline exhibits almost a power law scaling with statistics, with the best absolute performance in all cases for all luminosities in the upper and

lower rows. For the shorter baselines, the performance depends crucially on the true $\delta_{\mathrm{CP}}$. For $\delta_{\mathrm{CP}} \simeq \pi / 2$, the scaling behaves similar to the magic baseline, and there are only very few jumps. That means that changes in the chosen standard luminosity (scaling factor one) do not have a strong effect. Only for the ${ }^{18} \mathrm{Ne}$ and ${ }^{6} \mathrm{He}$ combination with very high $\gamma$, increasing the luminosity resolves the (wrong hierarchy) intrinsic degeneracy and this improves the performance of the setup such that the sensitivities approach the ones obtained for the magic baseline. For $\delta_{\mathrm{CP}} \simeq 3 \pi / 2$, the mass hierarchy sensitivity is basically not present for ${ }^{8} \mathrm{~B}$ and ${ }^{8} \mathrm{Li}$, and for ${ }^{18} \mathrm{Ne}$ and ${ }^{6} \mathrm{He}$ for $\gamma \ll 2000$. In summary, for the mass hierarchy sensitivity, the magic baseline is a safe choice, independent of the luminosity. Similar sensitivity can be achieved by the shorter baseline only for extreme choices of $\gamma$ and $N_{\beta}$.

\subsection{The CP Sensitivity Reach}

The $\sin ^{2} 2 \theta_{13}$ reach for the sensitivity to maximal CP violation $(3 \sigma)$ as a function of the luminosity scaling factor is shown in Fig. 11. The luminosity scaling factor multiplies the overall luminosities (useful isotope decays $\times$ running time $\times$ detector mass $\times$ detector efficiency) for both neutrinos and antineutrinos. The panels represent the different isotopes and different $\gamma$ values as given in the captions. For all curves, the short baselines are used, i.e., $L / \gamma=2.6$ for the upper row, and $L / \gamma=0.8$ for the middle and lower rows, because there is no CP violation sensitivity at the magic baseline. The solid curves are computed for $\delta_{\mathrm{CP}}$ (true) $=\pi / 2$, the dashed curves for $\delta_{\mathrm{CP}}($ true $)=3 \pi / 2$.

The scalings are very similar to the mass hierarchy sensitivity: For $\delta_{\mathrm{CP}} \simeq \pi / 2$ there are no jumps, and for $\delta_{\mathrm{CP}} \simeq 3 \pi / 2$ the intrinsic degeneracy can be resolved above a certain threshold luminosity (because it is then lifted over the $\chi^{2}=9$ line). All curves look qualitatively very similar, no matter what $\gamma$ or isotope is used. However, The absolute performance is better for ${ }^{18} \mathrm{Ne}+{ }^{6} \mathrm{He}$ even for the same $\gamma$.

As far as the chosen reference luminosity (scaling factor one) is concerned, the ${ }^{8} \mathrm{~B}$ and ${ }^{8} \mathrm{Li}$ beam is operated in a region where substantial luminosity changes will not affect the result. However, for ${ }^{18} \mathrm{Ne}$ and ${ }^{6} \mathrm{He}$, already a factor of two to three luminosity increase would be sufficient to resolve the intrinsic degeneracy and to boost the performance at $\delta_{\mathrm{CP}}$ (true) $=3 \pi / 2$ for the lower row. Note that such a boost could also be achieved by a synergistic degeneracy resolver, such as the magic baseline as second baseline. 

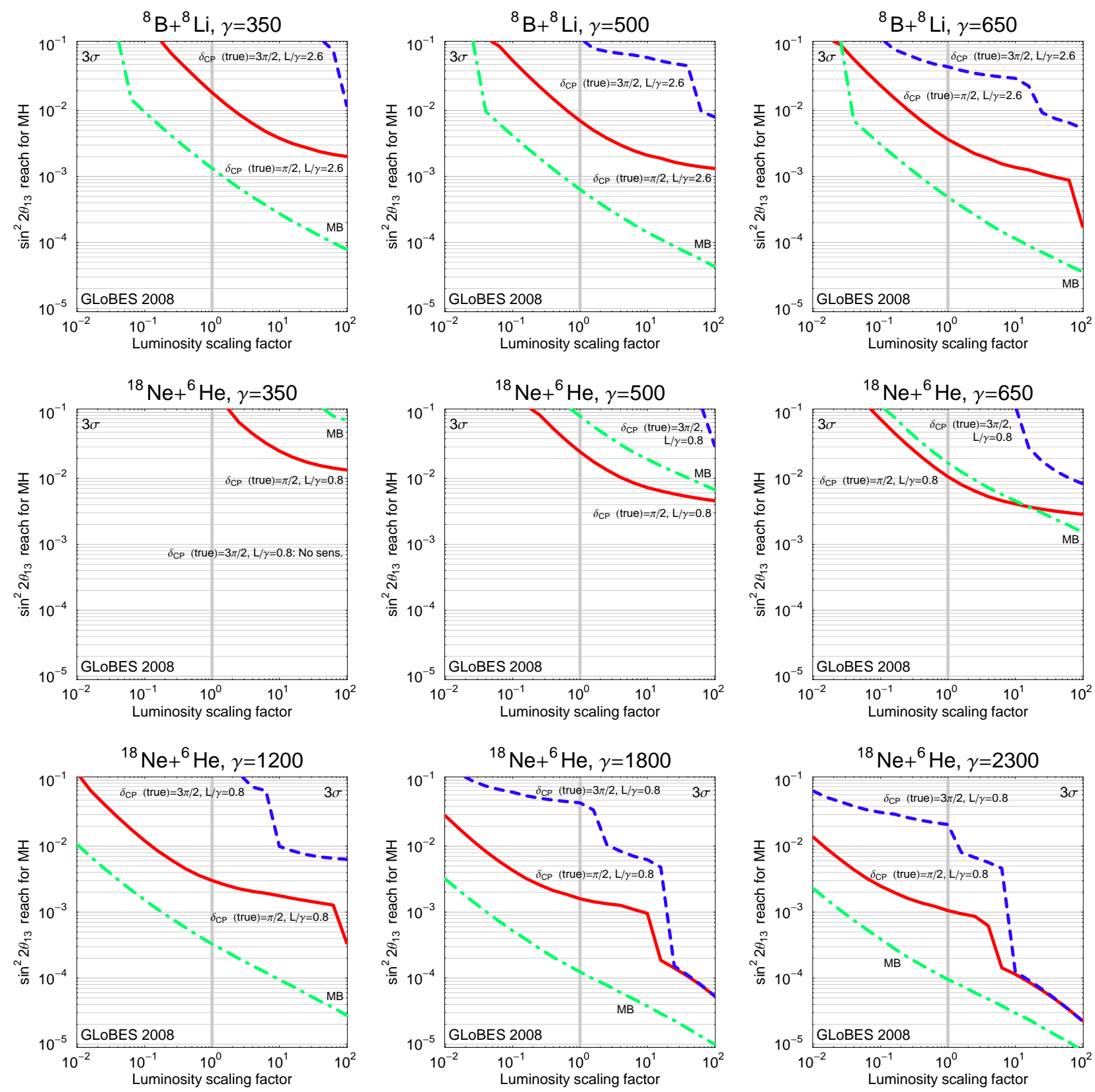

Figure 10: The $\sin ^{2} 2 \theta_{13}$ reach for the sensitivity to the normal mass hierarchy $(3 \sigma)$ as a function of a luminosity scaling factor. The luminosity scaling factor multiplies the overall luminosities (useful isotope decays $\times$ running time $\times$ detector mass $\times$ detector efficiency) for both neutrinos and antineutrinos. The panels represent the different isotopes and different $\gamma$ as indicated in the captions. The green dashed-dotted curves correspond to the long magic baseline "MB" with $L=7500 \mathrm{~km}$ fixed and very little dependence on the true $\delta_{\mathrm{CP}}$, the other two sets of curves to a short baseline with an $L / \gamma$ depending on the isotope (c.f., lines for fixed $L / \gamma$ in Fig. 6). The red solid curves are computed for a true $\delta_{\mathrm{CP}}=\pi / 2$, the blue dashed curves for a true $\delta_{\mathrm{CP}}=3 \pi / 2$. 

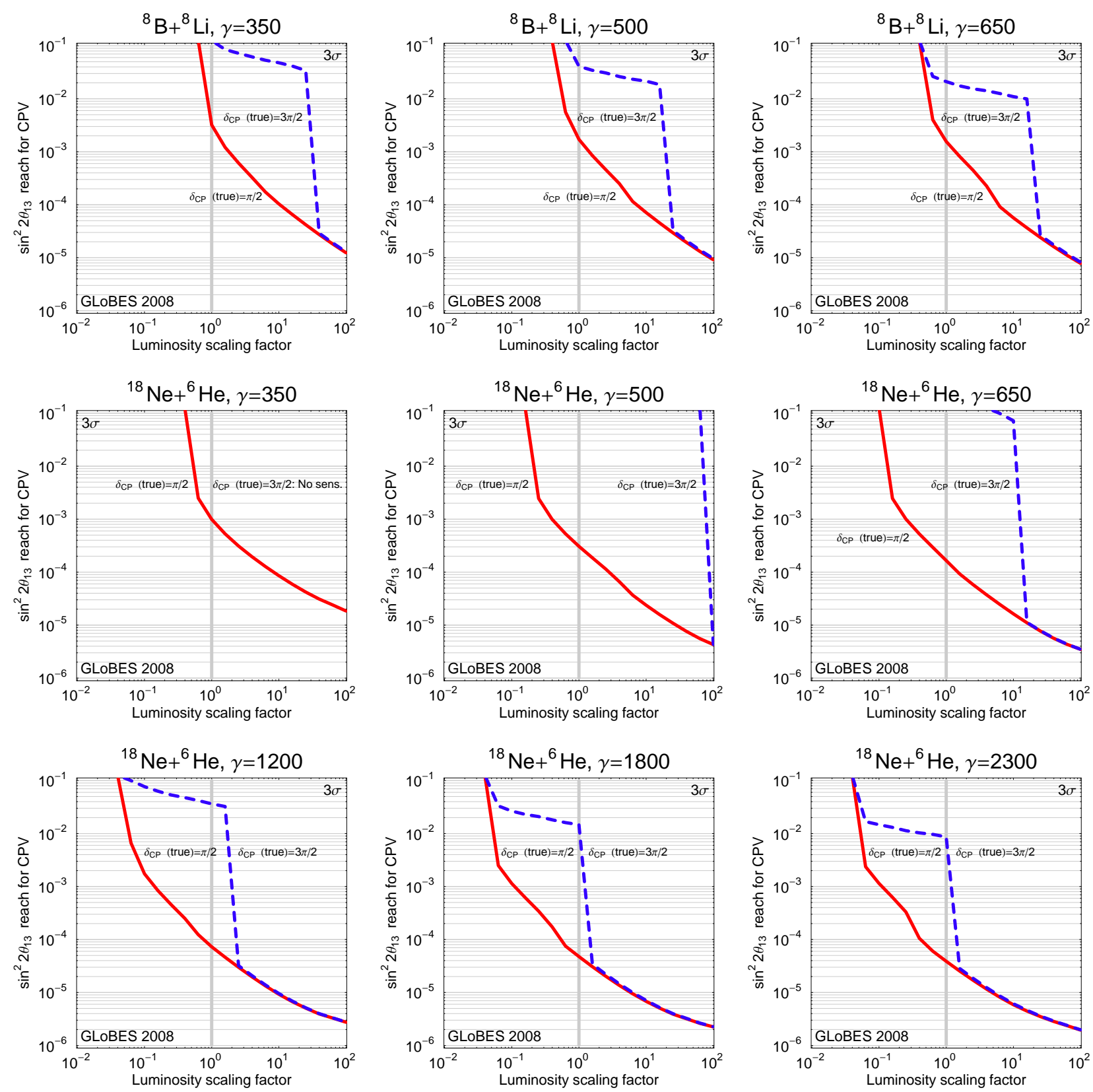

Figure 11: The $\sin ^{2} 2 \theta_{13}$ reach for the sensitivity to maximal CP violation $(3 \sigma)$ as a function of a luminosity scaling factor. The luminosity scaling factor multiplies the overall luminosities (useful isotope decays $\times$ running time $\times$ detector mass $\times$ detector efficiency) for both neutrinos and antineutrinos. The panels represent the different isotopes and different $\gamma$ as given in the captions. For all curves, the short baselines are used, i.e., $L / \gamma=2.6$ for the upper row, and $L / \gamma=0.8$ for the middle and lower rows. The red solid curves are computed for a true $\delta_{\mathrm{CP}}=\pi / 2$, the blue dashed curves for a true $\delta_{\mathrm{CP}}=3 \pi / 2$. A true normal hierarchy is assumed. 


\section{Summary and Conclusions}

Beta-beams provide intense and well understood neutrino fluxes of a single flavor. They have very low beam-related backgrounds and very low systematic uncertainties, and are hence ideal for precision experiments. While detailed physics reach analyses of specific projects involving Beta-beams have been performed in the literature, greenfield setups have been less extensively studied. In this study, we have considered the Beta-beam option as a greenfield scenario and have identified the optimal set-ups for an iron calorimeter as the detector. We have optimized for maximal reaches in $\sin ^{2} 2 \theta_{13}$, i.e., our Beta-beams are designed to measure $\sin ^{2} 2 \theta_{13}$, CP violation, and the mass hierarchy for as small $\sin ^{2} 2 \theta_{13}$ as possible. We have studied two pairs of plausible source ions for the Beta-beam: the standard ions ${ }^{18} \mathrm{Ne}$ and ${ }^{6} \mathrm{He}$, which have been extensively studied in the literature, as well as the new candidates ${ }^{8} \mathrm{~B}$ and ${ }^{8} \mathrm{Li}$. For each pair of source ions, we have optimized the experimental set-up as a function of baseline $L$, boost factor $\gamma$, and luminosity. The luminosity is proportional to the number of useful ion decays, detector efficiency, size of detector, and exposure time. We have followed two complementary approaches for our study: (i) Fixing the input parameters of the beam and looking for the sensitivity reach between the two pairs of ions as a function of the baseline. (ii) Matching the shape (energies) of the event spectrum and luminosity for the two sets of ions and studying the required input parameters of the beam as a function of the baseline. In both approaches, we have compared the physics reach for the two pairs of ions in order to identify the optimal conditions for the sensitivity reach.

In order to compare the two different pairs of isotopes, it has been useful to determine the conditions for the same physics output, i.e., neutrino energies, matter effects, etc., at the same baseline. Since the total flux at the detector for a fixed baseline is proportional to $N_{\beta} \gamma^{2}$ (with $N_{\beta}$ being the useful ion decays per year) and the peak neutrino energy is approximately given by $\gamma E_{0}$, we have identified the conditions for obtaining similar neutrino spectral shape as $N_{\beta}^{B+L i} \simeq$ $12 \cdot N_{\beta}^{N e+H e}, \gamma^{N e+H e} \simeq 3.5 \cdot \gamma^{B+L i}$. This means that, because of the stronger forward collimation of the beam, a smaller required $\gamma$ for ${ }^{8} \mathrm{~B}$ and ${ }^{8} \mathrm{Li}$ has, in principle, to be compensated by a correspondingly larger number of useful ion decays. We have verified this behavior in realistic simulations.

As the next step, we have fixed the beam input parameters $N_{\beta}$ to $1.1 \times 10^{18}\left(\nu_{e}\right)$ and $2.9 \times 10^{18}$ $\left(\bar{\nu}_{e}\right)$ useful ion decays/year, and $\gamma$ to 350, 500, and 650, respectively. Because of the higher neutrino energies for the same $\gamma,{ }^{8} \mathrm{~B}$ and ${ }^{8} \mathrm{Li}$ experience stronger matter effects than ${ }^{6} \mathrm{He}$ and ${ }^{18} \mathrm{Ne}$. This has two major implications: First, if the matter resonance energy can be covered, the $1 / L^{2}$ dependence of the events is reduced for ${ }^{8} \mathrm{~B}$ and ${ }^{8} \mathrm{Li}$, and the event rates increase at longer baselines. Second, because of the matter resonant or anti-resonant behavior, the mass hierarchy can be much easily determined. Both implications make the magic baseline attractive for the $\sin ^{2} 2 \theta_{13}$ and mass hierarchy measurements using ${ }^{8} \mathrm{~B}$ and ${ }^{8} \mathrm{Li}$. For ${ }^{6} \mathrm{He}$ and ${ }^{18} \mathrm{Ne}$, on the other hand, a much shorter baseline is preferable. For $\gamma>500,{ }^{8} \mathrm{~B}$ and ${ }^{8} \mathrm{Li}$ even outperform the ${ }^{6} \mathrm{He}$ and ${ }^{18} \mathrm{Ne}$ pair for the two discussed performance indicators. For CP violation however, the ${ }^{6} \mathrm{He}$ and ${ }^{18} \mathrm{Ne}$ turns out to have a discovery reach about one order of magnitude better than for ${ }^{8} \mathrm{~B}$ and ${ }^{8} \mathrm{Li}$ at a relatively short baseline $L \simeq 600-1000 \mathrm{~km}$ (depending on the $\gamma$ chosen). Similar to the neutrino factory, we therefore observe a tension between measuring the mass hierarchy (preferring long baselines and high energies close to the matter resonance energy), and measuring $\mathrm{CP}$ violation (preferring short baselines determined by the oscillation maximum with as little matter effects as possible). 
Therefore, in order to optimally access all performance indicators, one may finally require both pairs of ions and two baselines.

If one wants to compare similar neutrino energies for the two isotope pairs, i.e., one fixes the output, one has to include $\gamma^{\prime}$ s about a factor of three higher for ${ }^{6} \mathrm{He}$ and ${ }^{18} \mathrm{Ne}$ than for ${ }^{8} \mathrm{~B}$ and ${ }^{8} \mathrm{Li}$. From an optimization of our performance indicators in the $L-\gamma$ plane (while still keeping the useful ion decays fixed), we have learned that there are, in principle, two sets of baselines which exhibit local optima in the performances:

- A short baseline with $L[\mathrm{~km}] / \gamma \simeq 0.8$ for ${ }^{6} \mathrm{He}$ and ${ }^{18} \mathrm{Ne}$, and $L[\mathrm{~km}] / \gamma \simeq 2.6$ for ${ }^{8} \mathrm{~B}$ and ${ }^{8} \mathrm{Li}$.

- A long "magic" baseline $L \simeq 7500 \mathrm{~km}$, where the dependence on $\delta_{\mathrm{CP}}$ vanishes.

While the shorter baseline is always the better choice for the CP violation measurement, the choice between the longer and shorter baseline for the $\sin ^{2} 2 \theta_{13}$ sensitivity depends on $\gamma$ and the isotope used. For ${ }^{8} \mathrm{~B}$ and ${ }^{8} \mathrm{Li}$, the magic baseline is preferred for $\gamma>350$, while for ${ }^{6} \mathrm{He}$ and ${ }^{18} \mathrm{Ne}$, the shorter baseline is always preferred for any realistic $\gamma$ because of the higher event rates for the same neutrino energies. For the mass hierarchy sensitivity, the magic baseline tends to be the best choice for ${ }^{8} \mathrm{~B}$ and ${ }^{8} \mathrm{Li}$ already for $\gamma>300$, while for ${ }^{6} \mathrm{He}$ and ${ }^{18} \mathrm{Ne}$ a relatively high $\gamma>1000$ is required. However, in the latter case, the short baseline alone will not be sufficient to measure the mass hierarchy, another longer baseline is required.

As the last step, we have focused on the above two sets of baselines, and we have varied the luminosity. For the $\sin ^{2} 2 \theta_{13}$ sensitivity, we have demonstrated that the choice between the short and long baseline depends on the reference luminosity as well. For the mass hierarchy sensitivity, the conclusion to use a longer baseline is very robust. And for the CP violation sensitivity, which is only present at the short baseline, the luminosity plays a crucial role to resolve (otherwise present) degeneracies for $\delta_{\mathrm{CP}}=3 \pi / 2$ (for the normal hierarchy) or $\delta_{\mathrm{CP}}=\pi / 2$ (for the inverted hierarchy). Note that this degeneracy resolution could also be achieved with the combination of two baselines.

We conclude that a greenfield Beta-beam could have excellent sensitivity reaches for the $\sin ^{2} 2 \theta_{13}$, mass hierarchy, and CP violation discoveries. Comparison of the physics reach between $\left({ }^{8} \mathrm{~B},{ }^{8} \mathrm{Li}\right)$ and $\left({ }^{6} \mathrm{He},{ }^{18} \mathrm{Ne}\right)$ pairs is not at all straightforward. On the one hand, ${ }^{8} \mathrm{~B}$ and ${ }^{8} \mathrm{Li}$ produce a given neutrino energy by a boost factor about 3.5 times lower than that needed for ${ }^{18} \mathrm{Ne}$ and ${ }^{6} \mathrm{He}$. Therefore, the first pair of ions would be the preferred choice if one needed higher energy Beta-beams within the constraint of the envisaged accelerator facilities. On the other hand, lower boost factors result in a lower beam collimation, and hence lower statistics, which would have to be compensated by a higher luminosity. For ${ }^{18} \mathrm{Ne}$ and ${ }^{6} \mathrm{He}$ one would need a $\gamma$ about 3.5 times larger than for ${ }^{8} \mathrm{~B}$ and ${ }^{8} \mathrm{Li}$ to obtain the same neutrino energies. This constraint stretches the demanded $\gamma$ to the prohibitively large regime. The statistics, in return, would be about a factor of 10 higher. Therefore, the optimal selection of ions and baselines crucially depends on the boost factor and luminosity used, as well as the chosen detector technology. For our reference luminosity and iron detector, if the Beta-beam is operated at a realistically "high" $\gamma \sim 500$, one would optimally use ${ }^{18} \mathrm{Ne}$ and ${ }^{6} \mathrm{He}$ at the short baseline for $\mathrm{CP}$ violation, ${ }^{8} \mathrm{~B}$ and ${ }^{8} \mathrm{Li}$ at the magic baseline for mass hierarchy, and either ${ }^{18} \mathrm{Ne}$ and ${ }^{6} \mathrm{He}$ at the short baseline or ${ }^{8} \mathrm{~B}$ and ${ }^{8} \mathrm{Li}$ at the magic baseline for 
$\sin ^{2} 2 \theta_{13}$ discovery.

\section{Acknowledgments}

The authors acknowledge the HRI cluster facilities for computation. This work has been supported by the Neutrino Project under the XI Plan of Harish-Chandra Research Institute. W.W. would like to acknowledge support from Emmy Noether program of Deutsche Forschungsgemeinschaft, and through DFG grant 446 IND 111/9/07. In addition, he would like to thank the members of HRI for their warm hospitality during his stay.

\section{References}

[1] B. T. Cleveland et al., Astrophys. J. 496, 505 (1998); J. N. Abdurashitov et al. [SAGE Collaboration], J. Exp. Theor. Phys. 95, 181 (2002) [Zh. Eksp. Teor. Fiz. 122, 211 (2002)]; W. Hampel et al. [GALLEX Collaboration], Phys. Lett. B 447, 127 (1999); S. Fukuda et al. [Super-Kamiokande Collaboration], Phys. Lett. B 539, 179 (2002); B. Aharmim et al. [SNO Collaboration], Phys. Rev. C 72, 055502 (2005); C. Arpesella et al. [Borexino Collaboration], Phys. Lett. B 658, 101 (2008).

[2] T. Araki et al. [KamLAND Collaboration], Phys. Rev. Lett. 94, 081801 (2005); S. Abe et al. [KamLAND Collaboration], arXiv:0801.4589 [hep-ex].

[3] Y. Ashie et al. [Super-Kamiokande Collaboration], Phys. Rev. D 71, 112005 (2005).

[4] M. Apollonio et al., Eur. Phys. J. C 27, 331 (2003).

[5] E. Aliu et al. [K2K Collaboration], Phys. Rev. Lett. 94, 081802 (2005).

[6] D. G. Michael et al., [MINOS Collaboration], arXiv:hep-ex/0607088.

[7] M. C. Gonzalez-Garcia and M. Maltoni, arXiv:0704.1800 [hep-ph]; M. Maltoni, T. Schwetz, M. A. Tortola and J. W. F. Valle, New J. Phys. 6, 122 (2004), hep-ph/0405172 v5; S. Choubey, arXiv:hep-ph/0509217; S. Goswami, Int. J. Mod. Phys. A 21, 1901 (2006); A. Bandyopadhyay, S. Choubey, S. Goswami, S. T. Petcov and D. P. Roy, Phys. Lett. B 608, 115 (2005); G. L. Fogli et al., Prog. Part. Nucl. Phys. 57, 742 (2006).

[8] A. Cervera, A. Donini, M. B. Gavela, J. J. Gómez-Cadenas, P. Hernandez, O. Mena and S. Rigolin, Nucl. Phys. B 579, 17 (2000) [Erratum-ibid. B 593, 731 (2001)].

[9] P. Aprili et al. [ICARUS Collaboration], CERN-SPSC-2002-027 (2002).

[10] M. Guler et al. [OPERA Collaboration], CERN-SPSC-2000-028 (2000);

[11] Y. Itow et al., arXiv:hep-ex/0106019.

[12] D. S. Ayres et al. [NOvA Collaboration], arXiv:hep-ex/0503053. 
[13] F. Ardellier et al., arXiv:hep-ex/0405032; F. Ardellier et al. [Double Chooz Collaboration], arXiv:hep-ex/0606025.

[14] P. Huber, M. Lindner, M. Rolinec, T. Schwetz and W. Winter, Phys. Rev. D 70, 073014 (2004) and references therein.

[15] P. Zucchelli, Phys. Lett. B 532, 166 (2002).

[16] M. Lindroos, arXiv:physics/0312042; M. Lindroos, Nucl. Phys. Proc. Suppl. 155, 48 (2006).

[17] http://beta-beam.web.cern.ch/beta\%2Dbeam/

[18] S. Geer, Phys. Rev. D 57, 6989 (1998) [Erratum-ibid. D 59, 039903 (1999)].

[19] A. Cervera, Talk at NuFact07, Okayama University, Okayama, Japan, August 6-11, 2007, http://fphy.hep.okayama-u.ac.jp/nufact07/

[20] P. Huber, M. Mezzetto and T. Schwetz, arXiv:0711.2950 [hep-ph].

[21] http://www.hep.ph.ic.ac.uk/iss/

[22] A. Bandyopadhyay et al. [ISS Physics Working Group], arXiv:0710.4947 [hep-ph].

[23] J. Burguet-Castell, M. B. Gavela, J. J. Gómez-Cadenas, P. Hernandez and O. Mena, Nucl. Phys. B 608, 301 (2001).

[24] H. Minakata and H. Nunokawa, JHEP 0110, 001 (2001).

[25] G. L. Fogli and E. Lisi, Phys. Rev. D 54, 3667 (1996).

[26] V. Barger, D. Marfatia and K. Whisnant, Phys. Rev. D 65, 073023 (2002).

[27] H. Minakata and H. Nunokawa, Phys. Lett. B 413, 369 (1997); V. Barger, D. Marfatia and K. Whisnant, Phys. Rev. D 66, 053007 (2002); V. Barger, D. Marfatia and K. Whisnant, Phys. Lett. B 560, 75 (2003); O. Mena and S. J. Parke, Phys. Rev. D 70, 093011 (2004); O. Mena Requejo, S. Palomares-Ruiz and S. Pascoli, Phys. Rev. D 72, 053002 (2005); M. Ishitsuka, T. Kajita, H. Minakata and H. Nunokawa, Phys. Rev. D 72, 033003 (2005); K. Hagiwara, N. Okamura and K. i. Senda, Phys. Rev. D 76, 093002 (2007).

[28] P. Huber, M. Lindner and W. Winter, Nucl. Phys. B 645, 3 (2002); P. Huber, M. Lindner and W. Winter, Nucl. Phys. B 654, 3 (2003).

[29] A. Donini, D. Meloni and P. Migliozzi, Nucl. Phys. B 646, 321 (2002); D. Autiero et al., Eur. Phys. J. C 33, 243 (2004).

[30] A. Donini, E. Fernandez-Martinez and S. Rigolin, Phys. Lett. B 621, 276 (2005); A. Donini, E. Fernandez-Martinez, D. Meloni and S. Rigolin, Nucl. Phys. B 743, 41 (2006).

[31] S. K. Agarwalla, S. Choubey, S. Goswami and A. Raychaudhuri, Phys. Rev. D 75, 097302 (2007). 
[32] P. Huber, M. Maltoni and T. Schwetz, Phys. Rev. D 71, 053006 (2005);

[33] J. E. Campagne, M. Maltoni, M. Mezzetto and T. Schwetz, JHEP 0704, 003 (2007).

[34] P. Huber, M. Lindner, T. Schwetz and W. Winter, Nucl. Phys. B 665, 487 (2003).

[35] P. Huber and W. Winter, Phys. Rev. D 68, 037301 (2003).

[36] A. Y. Smirnov, arXiv:hep-ph/0610198.

[37] M. Freund, M. Lindner, S. T. Petcov and A. Romanino, Nucl. Phys. B 578, 27 (2000).

[38] P. Huber, M. Lindner, M. Rolinec and W. Winter, Phys. Rev. D 74, 073003 (2006).

[39] S. K. Agarwalla, A. Raychaudhuri and A. Samanta, Phys. Lett. B 629, 33 (2005).

[40] S. K. Agarwalla, S. Choubey and A. Raychaudhuri, Nucl. Phys. B 771, 1 (2007).

[41] S. K. Agarwalla, S. Choubey and A. Raychaudhuri, arXiv:0711.1459 [hep-ph].

[42] M. Mezzetto, J. Phys. G 29, 1771 (2003) [arXiv:hep-ex/0302007]. M. Mezzetto, Nucl. Phys. Proc. Suppl. 143, 309 (2005). M. Mezzetto, Nucl. Phys. Proc. Suppl. 155, 214 (2006);

[43] A. Donini, E. Fernandez-Martinez, P. Migliozzi, S. Rigolin and L. Scotto Lavina, Nucl. Phys. B 710, 402 (2005).

[44] A. Donini, E. Fernandez, P. Migliozzi, S. Rigolin, L. Scotto Lavina, T. Tabarelli de Fatis and F. Terranova, arXiv:hep-ph/0511134; A. Donini, E. Fernandez-Martinez, P. Migliozzi, S. Rigolin, L. Scotto Lavina, T. Tabarelli de Fatis and F. Terranova, Eur. Phys. J. C 48, 787 (2006).

[45] P. Coloma, A. Donini, E. Fernandez-Martinez and J. Lopez-Pavon, arXiv:0712.0796 [hep-ph].

[46] J. Burguet-Castell, D. Casper, E. Couce, J. J. Gómez-Cadenas and P. Hernandez, Nucl. Phys. B 725, 306 (2005). J. Burguet-Castell, D. Casper, J. J. Gómez-Cadenas, P. Hernandez and F. Sanchez, Nucl. Phys. B 695, 217 (2004).

[47] A. Jansson, O. Mena, S. Parke and N. Saoulidou, arXiv:0711.1075 [hep-ph].

[48] P. Huber, M. Lindner, M. Rolinec and W. Winter, Phys. Rev. D 73, 053002 (2006).

[49] C. Volpe, J. Phys. G 34, R1 (2007).

[50] A. Donini and E. Fernandez-Martinez, Phys. Lett. B 641, 432 (2006).

[51] R. Adhikari, S. K. Agarwalla and A. Raychaudhuri, Phys. Lett. B 642, 111 (2006). S. K. Agarwalla, S. Rakshit and A. Raychaudhuri, Phys. Lett. B 647, 380 (2007).

[52] D. Meloni, O. Mena, C. Orme, S. Palomares-Ruiz and S. Pascoli, arXiv:0802.0255 [hep-ph]. 
[53] C. Rubbia, A. Ferrari, Y. Kadi and V. Vlachoudis, Nucl. Instrum. Meth. A 568, 475 (2006);

C. Rubbia, arXiv:hep-ph/0609235.

[54] Y. Mori, Nucl. Instrum. Meth. A 562, 591 (2006).

[55] M. S. Athar et al. [INO Collaboration], A Report of the INO Feasibility Study, http://www.imsc.res.in/ ino/OpenReports/INOReport.pdf

[56] S. Choubey, Talk at NuFact07, Okayama University, Okayama, Japan, August 6-11, 2007, http://fphy.hep.okayama-u.ac.jp/nufact07/; S. K. Agarwalla, S. Choubey and A. Raychaudhuri, arXiv:0712.4072 [hep-ph].

[57] B. Autin, R. C. Fernow, S. Machida and D. A. Harris, J. Phys. G 29, 1637 (2003); F. Terranova, A. Marotta, P. Migliozzi and M. Spinetti, Eur. Phys. J. C 38, 69 (2004).

[58] L. P. Ekstrom and R. B. Firestone, WWW Table of Radioactive Isotopes, database version 2/28/99 from URL http://ie.lbl.gov/toi/

[59] M. Lindroos, Talk at the "First plenary meeting of the International Design Study for the Neutrino Factory", RAL, United Kingdom, 16-17 January 2008.

[60] C. K. Jung, AIP Conf. Proc. 533, 29 (2000)

[61] Y. Itow et al., arXiv:hep-ex/0106019.

[62] A. de Bellefon et al., arXiv:hep-ex/0607026.

[63] L. Wolfenstein, Phys. Rev. D 17, 2369 (1978);

[64] S. P. Mikheev and A. Y. Smirnov, Sov. J. Nucl. Phys. 42, 913 (1985) [Yad. Fiz. 42, 1441 (1985)]; S. P. Mikheev and A. Y. Smirnov, Nuovo Cim. C 9, 17 (1986).

[65] V. D. Barger, K. Whisnant, S. Pakvasa and R. J. N. Phillips, Phys. Rev. D 22, 2718 (1980).

[66] M. Freund, P. Huber and M. Lindner, Nucl. Phys. B 615, 331 (2001).

[67] A. M. Dziewonski and D. L. Anderson, Phys. Earth Planet. Interiors 25, 297 (1981);

S. V. Panasyuk, Reference Earth Model (REM) webpage, http://cfauves5.harvrd.edu/lana/rem/index.html.

[68] See for example, A. Bandyopadhyay, S. Choubey, S. Goswami and S. T. Petcov, Phys. Rev. D 72, 033013 (2005); J. N. Bahcall and C. Pena-Garay, JHEP 0311, 004 (2003).

[69] R. J. Geller and T. Hara, Nucl. Instrum. Meth. A 503, 187 (2001).

[70] P. Huber, J. Kopp, M. Lindner, M. Rolinec and W. Winter, Comput. Phys. Commun. 177, 432 (2007); P. Huber, M. Lindner and W. Winter, Comput. Phys. Commun. 167, 195 (2005).

[71] M. Lindroos, Talk at Moriond Workshop on "Radioactive beams for nuclear physics and neutrino physics" March 17-22nd, 2003; http://moriond.in2p3.fr/radio/index.html. 